\title{
One-step ahead forecasting of geophysical processes within a purely statistical framework
}

\author{
Georgia Papacharalampous*, Hristos Tyralis and Demetris Koutsoyiannis
}

\begin{abstract}
The simplest way to forecast geophysical processes, an engineering problem with a widely recognized challenging character, is the so-called "univariate time series forecasting" that can be implemented using stochastic or machine learning regression models within a purely statistical framework. Regression models are in general fast-implemented, in contrast to the computationally intensive Global Circulation Models, which constitute the most frequently used alternative for precipitation and temperature forecasting. For their simplicity and easy applicability, the former have been proposed as benchmarks for the latter by forecasting scientists. Herein, we assess the one-step ahead forecasting performance of 20 univariate time series forecasting methods, when applied to a large number of geophysical and simulated time series of 91 values. We use two real-world annual datasets, a dataset composed by 112 time series of precipitation and another composed by 185 time series of temperature, as well as their respective standardized datasets, to conduct several real-world experiments. We further conduct large-scale experiments using 12 simulated datasets. These datasets contain 24,000 time series in total, which are simulated using stochastic models from the families of AutoRegressive Moving Average and AutoRegressive Fractionally Integrated Moving Average. We use the first 50, 60, 70, 80 and 90 data points for model-fitting and model-validation, and make predictions corresponding to the $51 \mathrm{st}, 61 \mathrm{st}, 71 \mathrm{st}, 81 \mathrm{st}$ and $91 \mathrm{st}$ respectively. The total number of forecasts produced herein is 2,177,520, among which 47,520 are obtained using the real-world datasets. The assessment is based on eight error metrics and accuracy statistics. The simulation experiments reveal the most and least accurate methods for long-term forecasting applications, also suggesting that the simple methods may be competitive in specific cases. Regarding the results of the realworld experiments using the original (standardized) time series, the minimum and maximum medians of the absolute errors are found to be $68 \mathrm{~mm}(0.55)$ and $189 \mathrm{~mm}(1.42)$ respectively for precipitation, and $0.23{ }^{\circ} \mathrm{C}(0.33)$ and $1.10^{\circ} \mathrm{C}$ (1.46) respectively for temperature. Since there is an absence of relevant information in the literature, the numerical results obtained using the standardized real-world datasets could be used as rough benchmarks for the one-step ahead predictability of annual precipitation and temperature.
\end{abstract}

Keywords: ARFIMA, Benchmarking time series forecasts, Machine learning, Neural networks, Precipitation, Random forests, Simple exponential smoothing, Support vector machines, Temperature, Univariate time series forecasting

\section{Background}

Forecasting geophysical variables in various time scales and horizons is useful in technological applications (e.g. Giunta et al. 2015), but a difficult task as well.

\footnotetext{
*Correspondence: papacharalampous.georgia@gmail.com Department of Water Resources and Environmental Engineering, School of Civil Engineering, National Technical University of Athens, Iroon Polytechniou 5, 15780 Zografou, Greece
}

Precipitation and temperature forecasting is mostly based on deterministic models as the Global Circulation Models (GCMs), which simulate the Earth's atmosphere using numerical equations; therefore, deviating from traditional time series forecasting, i.e. univariate time series forecasting. This particular deviation has been questioned by forecasting scientists (Green and Armstrong 2007; Green et al. 2009; Fildes and Kourentzes 2011, see also the comments in Keenlyside 2011; McSharry 2011). 
Traditional time series forecasting can be performed using several classes of regression models, as reviewed in De Gooijer and Hyndman (2006), while the two major classes are stochastic and machine learning. Regression models are in general fast-implemented in contrast to their computationally intensive alternative in precipitation and temperature forecasting, i.e. the GCMs. For their simplicity and easy applicability, the former have been proposed as benchmarks for the latter by Green et al. (2009).

Recognizing the necessity of introducing traditional forecasting methods in temperature and precipitation forecasting, Armstrong and Fildes (2006) have recommended a relevant issue in one of the Journals specialized in forecasting. Since then and despite the fact that considerable parts of books in hydrology are devoted to such methods (Sivakumar 2017, pp 63-145; Remesan and Mathew 2015, pp 71-110), there has not been a systematic approach to the subject. However, studies adopting statistical forecasting approaches in geoscience are sporadically published in a variety of Journals. Within a statistical framework, Tyralis and Koutsoyiannis (2014, 2017) use Bayesian techniques for probabilistic climate forecasts under the established assumption of longrange dependence of the observed time series. In the latter study information from GCMs is used to improve the performance of the time series forecasting methods. Moreover, Table 1 presents some examples of studies using univariate time series forecasting approaches that do not utilize exogenous predictor variables to forecast precipitation or temperature variables, and streamflow or river discharge variables. The former can be considered as climatic or meteorological variables depending on the time scale of interest, while the latter can be considered as the results of precipitation (and other) variables and are more frequently modelled by describing this dependence using either deterministic or statistical methods. Such statistical approaches to modelling hydrological variables can be found in Chen et al. (2015), Gholami et al. (2015) and Taormina and Chau (2015).

In a somehow different direction, Papacharalampous et al. (2017c) conduct a multiple-case study, i.e. a synthesis of 50 single-case studies, by using monthly precipitation and temperature time series of various lengths observed in Greece. Some important points regarding the comparison of univariate time series forecasting methods and additional concerns introduced when implementing the machine learning ones (hyperparameter optimization and lagged variable selection) in oneand multi-step ahead forecasting are illustrated in the latter study. Nevertheless, only large-scale forecast-producing studies could provide empirical solutions to several problems appearing in the field of (geophysical) time series forecasting. Such studies are rare in the literature. Beyond geoscience, Makridakis and Hibon (2000) use a real-world dataset composed by 3003 time series, mainly originating from the business, industry, macroeconomic and microeconomic sectors, to assess the one- and multistep ahead forecasting accuracy of 24 univariate time series forecasting methods. In geoscience, on the other hand, there are only four recent studies, all companions of the present, to be subsequently discussed.

Papacharalampous et al. (2017a) compare 11 stochastic and nine machine learning univariate time series forecasting methods in multi-step ahead forecasting of geophysical processes and (empirically) prove that stochastic and machine learning methods can perform equally well. The comparisons are conducted using 24,000 simulated time series of 110 values, 24,000 simulated time series of 310 values and 92 mean monthly time series of streamflow with varying lengths, as well as 18 metrics. These 20 methods are also found to collectively compose a representative sample set, i.e. exhibiting a variety of forecasting performances with respect to the different metrics. Alongside with this study, Papacharalampous et al. (2017b) investigate the error evolution in multistep ahead forecasting when adopting this specific set of methods. The tests are performed on 6000 simulated time series of 150 values, 6000 simulated time series of 350 values and the streamflow dataset used in Papacharalampous et al. (2017a). Some different behaviours are revealed within these experiments, also suggesting the fact that one- and multi-step ahead forecasting are different problems to be examined for the same methods. Moreover, Tyralis and Papacharalampous (2017) focus on random forests, a well-known machine learning algorithm, with the aim to improve its one-step ahead forecasting performance by conducting experiments on 16,000 simulated and 135 annual temperature time series of 101 values. Finally, Papacharalampous et al. (2018) investigate the multi-step ahead predictability of monthly precipitation and temperature by applying seven automatic univariate time series forecasting methods to a sample of 1552 monthly precipitation and 985 monthly temperature time series of 480 values.

Herein, we examine the fundamental problem of onestep ahead forecasting, also complementing the results of the four above-mentioned studies. In more detail, we expand the former of these studies by exploring the onestep ahead forecasting properties of its methods, when applied to geophysical time series. Emphasis is put on the examination of two real-world datasets, a precipitation dataset and a temperature dataset, together containing 297 annual time series of 91 values. These datasets are examined in both their original and standardized forms. We further perform experiments using 24,000 simulated 
Table 1 Examples of univariate time series forecasting in geoscience

\begin{tabular}{|c|c|c|c|c|c|c|}
\hline$s / n$ & Study & Process & $\begin{array}{l}\text { Number of origi- } \\
\text { nal time series }\end{array}$ & Forecast time scale & $\begin{array}{l}\text { Forecast horizon(s) } \\
\text { [step(s) ahead] }\end{array}$ & $\begin{array}{l}\text { Univariate time series forecasting } \\
\text { method(s) }\end{array}$ \\
\hline 1 & Hong (2008) & Precipitation & 9 & Hourly & 1 & $\begin{array}{l}\text { (1) Support vector machines } \\
\text { (2) Hybrid model, i.e. a combination } \\
\text { of recurrent neural networks and } \\
\text { support vector machines }\end{array}$ \\
\hline 2 & Chau and Wu (2010) & & 2 & Daily & $1,2,3$ & $\begin{array}{l}\text { (1) Neural networks } \\
\text { (2) Hybrid model, i.e. a combination } \\
\text { of neural networks and support } \\
\text { vector machines }\end{array}$ \\
\hline 3 & $\begin{array}{l}\text { Htike and Khalifa } \\
\text { (2010) }\end{array}$ & & 1 & $\begin{array}{l}\text { Monthly, biannually, } \\
\text { quarterly, yearly }\end{array}$ & 1 & Neural networks \\
\hline 4 & Wu et al. (2010) & & 4 & Monthly, daily & $1,2,3$ & $\begin{array}{l}\text { (1) Linear regression } \\
\text { (2) } k \text {-nearest neighbours } \\
\text { (3) Neural networks } \\
\text { (4) Hybrid model, i.e. a combination } \\
\text { of neural networks }\end{array}$ \\
\hline 5 & $\begin{array}{l}\text { Narayanan et al. } \\
\text { (2013) }\end{array}$ & & 6 & Yearly & $21 \times 3$ (months) & $\begin{array}{l}\text { AutoRegressive Integrated Moving } \\
\text { Average (ARIMA) }\end{array}$ \\
\hline 6 & Wang et al. (2013) & & 1 & Monthly & 12 & $\begin{array}{l}\text { Seasonal AutoRegressive Integrated } \\
\text { Moving Average (SARIMA) }\end{array}$ \\
\hline 7 & $\begin{array}{l}\text { Babu and Reddy } \\
\text { (2012) }\end{array}$ & Temperature & 1 & Yearly & 10 & $\begin{array}{l}\text { (1) ARIMA } \\
\text { (2) Wavelet-based ARIMA }\end{array}$ \\
\hline 8 & $\begin{array}{l}\text { Chawsheen and } \\
\text { Broom (2017) }\end{array}$ & & 1 & Monthly & 121 & SARIMA \\
\hline 9 & Lambrakis et al. (2000) & $\begin{array}{l}\text { Streamflow } \\
\text { or river }\end{array}$ & 1 & Daily & 1 & $\begin{array}{l}\text { (1) Farmer's model } \\
\text { (2) Neural networks }\end{array}$ \\
\hline 10 & Ballini et al. (2001) & discharge & 1 & Monthly & $1,3,6,12$ & $\begin{array}{l}\text { (1) AutoRegressive Moving Average } \\
\text { (ARMA) } \\
\text { (2) Neural networks } \\
\text { (3) Neurofuzzy networks }\end{array}$ \\
\hline 11 & Yu et al. (2004) & & 2 & Daily & 1 & $\begin{array}{l}\text { (1) Support vector machines coupled } \\
\text { with an evolutionary algorithm } \\
\text { (2) Standard chaos technique } \\
\text { (3) Naïve } \\
\text { (4) Inverse approach } \\
\text { (5) ARIMA }\end{array}$ \\
\hline 12 & Komorník et al. (2006) & & 7 & Monthly & $1,3,6,12$ & $\begin{array}{l}\text { (1) Threshold AutoRegressive (AR) } \\
\text { with aggregation operators } \\
\text { (2) Logistic smooth transition AR } \\
\text { (3) Self-exciting threshold AR } \\
\text { (4) Naïve }\end{array}$ \\
\hline 13 & Yu and Liong (2007) & & 2 & Daily & 1 & $\begin{array}{l}\text { (1) Support vector machines coupled } \\
\text { with decomposition } \\
\text { (2) Standard chaos technique } \\
\text { (3) Naïve } \\
\text { (4) Inverse approach } \\
\text { (5) ARIMA }\end{array}$ \\
\hline 14 & $\begin{array}{l}\text { Koutsoyiannis et al. } \\
\text { (2008) }\end{array}$ & & $1 \times 12$ (months) & Yearly & 1 & $\begin{array}{l}\text { (1) Stochastic } \\
\text { (2) Analogue method } \\
\text { (3) Neural networks }\end{array}$ \\
\hline 15 & Wang et al. (2015) & & 3 & Monthly & 12 & SARIMA \\
\hline
\end{tabular}

time series of 91 values. These experiments complement the real-world ones by allowing the examination of a large variety of process behaviours, while they are also controlled to some extent, facilitating generalizations and increasing the understanding on the examined problem. The number of forecasts produced using these realworld and simulated datasets are 47,520 and 2,130,000, respectively, i.e. the largest among its companion studies. Our aim is twofold, to provide generalized results regarding one-step ahead forecasting within a purely statistical framework [justified, for example, in Hyndman and Athanasopoulos (2013)] in geoscience and hopefully to establish the results obtained by the examination of the standardized real-world datasets as rough benchmarks 
Table 2 Datasets of this study (part 1): real-world datasets

\begin{tabular}{lllllll}
\hline $\mathbf{s} \mathbf{n}$ & Abbreviated name & Process & Type & Primal dataset & R algorithm & Number of time series \\
\hline 1 & PrecDat & Precipitation & Original & Peterson and Vose (1997) & & 112 \\
2 & TempDat & Temperature & & Lawrimore et al. (2011) & 185 & mleHK \{HKprocess\} \\
3 & StandPrecDat & Precipitation & Standardized & PrecDat & 112 & 185 \\
4 & StandTempDat & Temperature & & TempDat & & 185
\end{tabular}

for the one-step ahead predictability of annual precipitation and temperature. The establishment of forecasting benchmarks is meaningful, especially for the one-step ahead attempts, as the latter constitute the most simple ones and their accuracy can be quantified using a single metric, i.e. the absolute error.

\section{Data and methods}

We use the datasets briefly described in Tables 2 and 3. The PrecDat and TempDat datasets are annual and originate from two larger monthly datasets, available in Peterson and Vose (1997) and Lawrimore et al. (2011) respectively. The sample period is from 1910 to 2000, so the following two conditions are simultaneously met: (1) there are no missing values, (2) the number of stations around the globe is the largest possible. We note that for sample periods extending after 2000 the number of retained stations would decrease rapidly. Figure 1 presents the maps of the retained stations. The precipitation ones create a sufficiently dense network in the United States of America and in Scandinavia, while the retained temperature stations in the United States of America, in Japan and in a part of South Korea. As it is apparent from Table 2, the StandPrecDat and StandTempDat datasets simply contain the standardized time series of PrecDat and TempDat respectively.
Figure 1 also presents the histograms of the Hurst parameter maximum likelihood estimates (Tyralis and Koutsoyiannis 2011) of the formed real-world time series. These estimates are of importance within this study for two reasons: (1) we implement a univariate time series forecasting method (see later on in this section) that takes advantage of this information under the established assumption of long-range dependence, (2) we standardize the original real-world time series using the mean and standard deviation maximum likelihood estimates (estimated simultaneously with the Hurst parameter) of the Hurst-Kolmogorov process. The standard deviation estimates would be considerably different if we modelled the time series using independent normal variables (Tyralis and Koutsoyiannis 2011). For consistency purposes with respect to the real-world datasets of the present study (but also to approximate the typical length of annual geophysical time series), the simulated time series are of 91 values as well. They originate from the families of AutoRegressive Moving Average $(\operatorname{ARMA}(p, q))$ and AutoRegressive Fractionally Integrated Moving Average $(\operatorname{ARFIMA}(p, d, q))$, the definitions of which can easily be found in the literature, for example in Wei (2006), pp 6-65, 489-494. The simulations are performed with mean 0 and standard deviation of 1 . Hereafter, to specify a used $\mathrm{R}$ algorithm, we state its name accompanied

Table 3 Datasets of this study (part 2): simulated datasets

\begin{tabular}{|c|c|c|c|c|c|}
\hline$s / n$ & Abbreviated name & Process & Parameter(s) & R algorithm & Number of time series \\
\hline 5 & SimDat_1 & $\operatorname{AR}(1)$ & $\varphi_{1}=0.7$ & arima.sim $\{$ stats $\}$ & 2000 \\
\hline 6 & SimDat_2 & $A R(1)$ & $\varphi_{1}=-0.7$ & & \\
\hline 7 & SimDat_3 & $\operatorname{AR}(2)$ & $\varphi_{1}=0.7, \varphi_{2}=0.2$ & & \\
\hline 8 & SimDat_4 & $M A(1)$ & $\theta_{1}=0.7$ & & \\
\hline 9 & SimDat_5 & $\mathrm{MA}(1)$ & $\theta_{1}=-0.7$ & & \\
\hline 10 & SimDat_6 & $\operatorname{ARMA}(1,1)$ & $\varphi_{1}=0.7, \theta_{1}=0.7$ & & \\
\hline 11 & SimDat_7 & $\operatorname{ARMA}(1,1)$ & $\varphi_{1}=-0.7, \theta_{1}=-0.7$ & & \\
\hline 12 & SimDat_8 & $\operatorname{ARFIMA}(0,0.30,0)$ & & fracdiff.sim $\{$ fracdiff $\}$ & \\
\hline 13 & SimDat_9 & $\operatorname{ARFIMA}(1,0.30,0)$ & $\varphi_{1}=0.7$ & & \\
\hline 14 & SimDat_10 & $\operatorname{ARFIMA}(0,0.30,1)$ & $\theta_{1}=-0.7$ & & \\
\hline 15 & SimDat_11 & $\operatorname{ARFIMA}(1,0.30,1)$ & $\varphi_{1}=0.7, \theta_{1}=-0.7$ & & \\
\hline 16 & SimDat_12 & $\operatorname{ARFIMA}(2,0.30,2)$ & $\varphi_{1}=0.7, \varphi_{2}=0.2, \theta_{1}=-0.7, \theta_{2}=-0.2$ & & \\
\hline
\end{tabular}




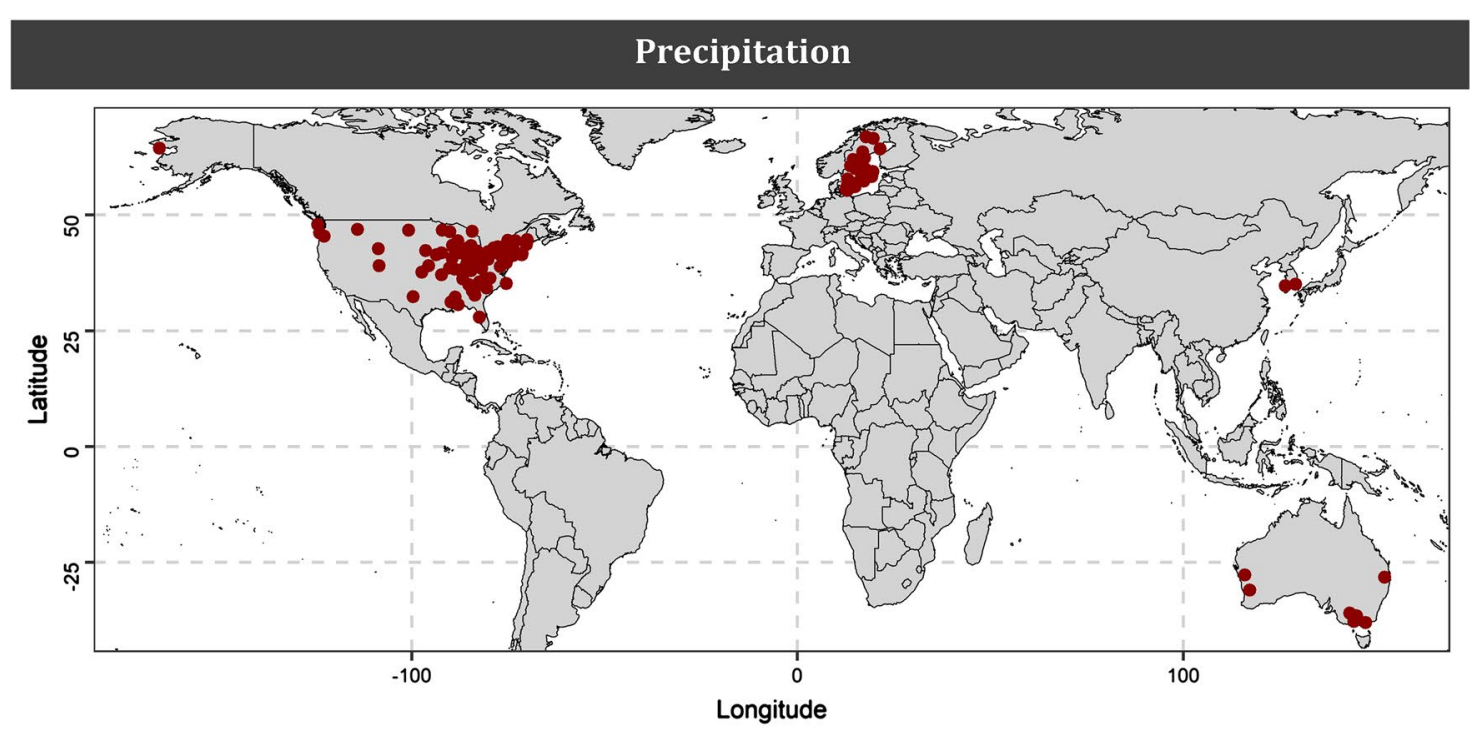

\section{Temperature}
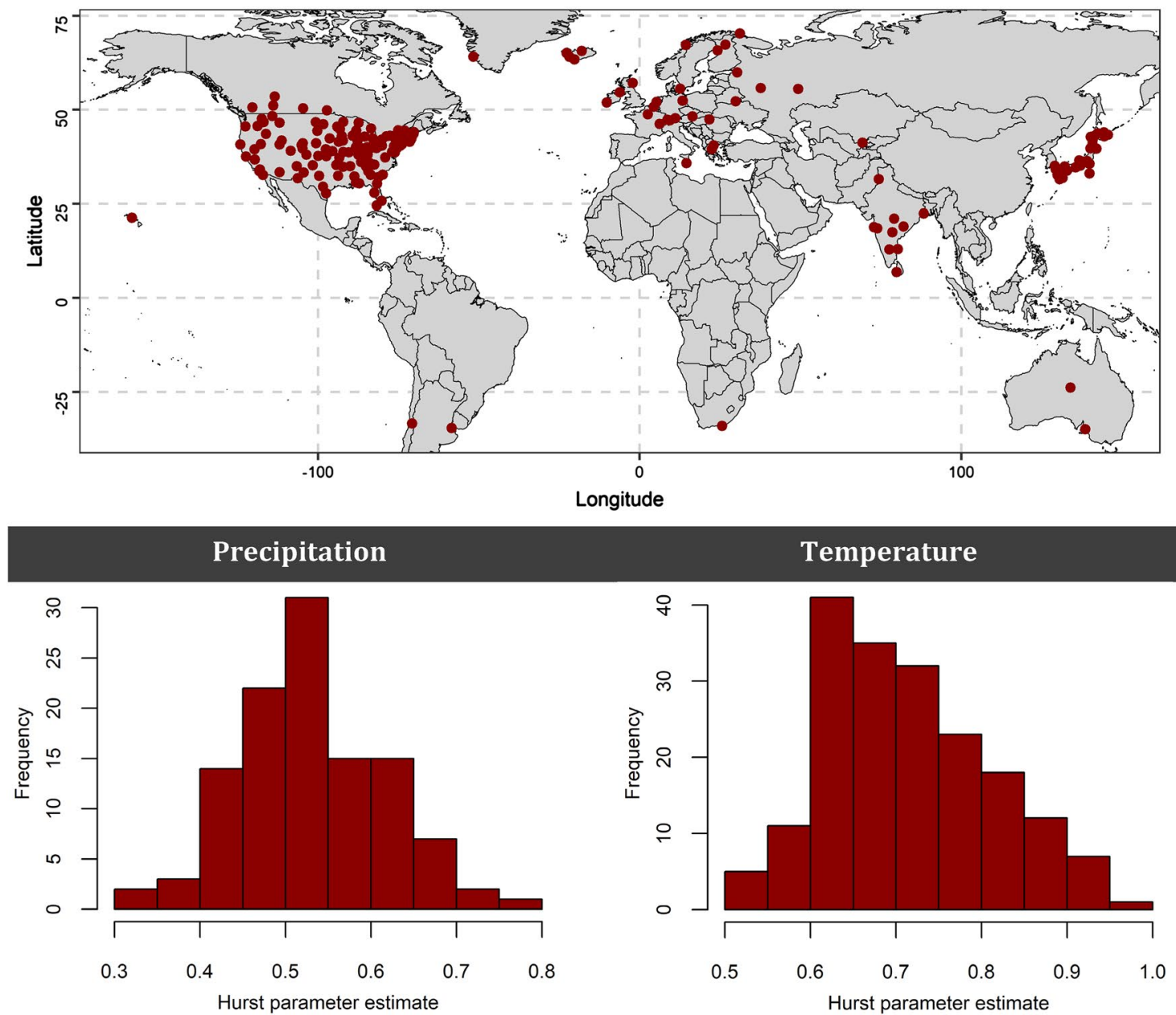

\section{Temperature}

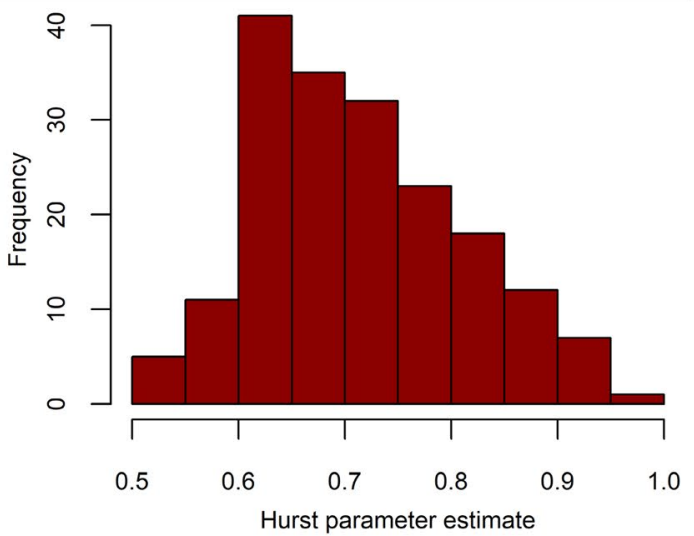

Fig. 1 Precipitation and temperature data from Peterson and Vose (1997) and Lawrimore et al. (2011) respectively 
by the name of the $\mathrm{R}$ package, denoted with \{\} . All algorithms are used with predefined values, unless specified differently.

We implement the forecasting methods described in Tables 4, 5 and 6 . The latter constitute an adapted reproduction from Papacharalampous et al. (2017a). Naïve is the last observation benchmark, while random walk (RW) is a commonly used variation of Naïve (Hyndman and Athanasopoulos 2013). Regarding the AutoRegressive Integrated Moving Average (ARIMA) methods, the ARIMA_f and auto_ARIMA_f forecasting models use the same algorithm with the ARIMA_s and auto_ARIMA_s simulation models respectively, although the innovations are set to be zero in the former ones. The latter applies to the auto_ARFIMA method as well, which is commonly used for modelling processes that are assumed to exhibit long-range dependence. These five methods estimate the involved parameters using the maximum likelihood method. BATS, ETS and SES stand for Box-Cox transformation, ARMA errors, Trend and Seasonal components (De Livera et al. 2011); Error, Trend and Seasonality (or ExponenTial Smoothing); and Simple Exponential Smoothing respectively. Further information about the latter models can be found in Hyndman and Athanasopoulos (2013), while Theta is introduced in Assimakopoulos and Nikolopoulos (2000). All the stochastic methods use procedures like those presented in Hyndman and Khandakar (2008). The machine learning methods, on the other hand, are based on a somehow different algorithmic approach. This fact is easily perceivable through the alongside examination of Tables 4, 5 and 6.

The assessment of the one-step ahead forecasting performance is based on the error metrics and accuracy statistics of Table 7 .

We conduct the experiments described in Tables 8 and 9. We use each dataset in five experiments; every time examining different parts of the time series according to Table 9. While the application of the stochastic methods does not require a validation set (since all the model parameters are estimated using other procedures, such as the maximum likelihood estimation), the same does not apply to the application of the machine learning methods (except NN_3). For each of the latter, we fit the candidate models defined in Table 5 to the fitting set, i.e. the first $33,40,47,53$ or 60 values, and subsequently use them to make predictions corresponding to the validation set, i.e. the next 17, 20, 23, 27 or 30 values respectively. Finally, we decide on the "optimal" model, i.e. the one exhibiting the smallest root mean square error on the validation set. We fit this model to the first $50,60,70,80$ or 90 values and make predictions corresponding to the 51st, 61st, 71 st, 81 st or 91 st value respectively.

The only assumption of our methodological approach concerns the application of the auto_ARFIMA method within the real-world experiments and is that the annual precipitation and temperature variables can be sufficiently modelled by the normal distribution. This assumption is rather reasonable (implied by the Central Limit Theorem; Koutsoyiannis 2008, chapter 2.5.6) and could hardly harm the results. In general, such fundamental assumptions are preferable to the introduction of extra parameters, e.g. to using the Box-Cox transformation to normalize the data. The rest of the methods are non-parametric and, thus, not affected by the possible non-normality. To take advantage of some well-known theoretical properties, in the $\mathrm{SE} \_1 i-\mathrm{SE}_{-} 7 i$ simulation experiments the ARIMA_f and ARIMA_s methods are given the same AutoRegressive (AR) and Moving Average (MA) orders used in the respective simulation process, while $d$ is set 0 . These two methods, as well as the simple, auto_ARIMA_f, auto_ARIMA_s and auto_ ARFIMA methods serve as reference points within our

Table 4 Univariate time series forecasting methods of this study (part 1): stochastic methods

\begin{tabular}{|c|c|c|c|c|}
\hline $\mathbf{s} / \mathbf{n}$ & Abbreviated name & Category & R algorithm(s) & Implementation notes \\
\hline 1 & Naïve & Simple & & \\
\hline 2 & RW & & rwf $\{$ forecast $\}$ & drift $=$ TRUE \\
\hline 3 & ARIMA_f & \multirow{4}{*}{$\begin{array}{l}\text { AutoRegressive Integrated Moving } \\
\text { Average (ARIMA) }\end{array}$} & Arima $\{$ forecast $\}$, forecast $\{$ forecast $\}$ & \multirow{4}{*}{$\begin{array}{l}\frac{\text { Arima }\{\text { forecast }\}}{\text { include.drift }}=\text { include. mean }=T R U E \text { FA, method }=" M L "\end{array}$} \\
\hline 4 & ARIMA_S & & Arima $\{$ forecast $\}$, simulate $\{$ stats $\}$ & \\
\hline 5 & auto_ARIMA_f & & auto.arima $\{$ forecast\}, forecast $\{$ forecast $\}$ & \\
\hline 6 & auto_ARIMA_s & & auto.arima $\{$ forecast\}, simulate $\{$ stats $\}$ & \\
\hline 7 & auto_ARFIMA & $\begin{array}{c}\text { AutoRegressive Fractionally Integrated } \\
\text { Moving Average (ARFIMA) }\end{array}$ & arfima \{forecast\}, forecast \{forecast\} & $\underline{\text { arfima }\{\text { forecast }\}}$ : estim = "mle" \\
\hline 8 & BATS & State space & bats $\{$ forecast $\}$, forecast $\{$ forecast $\}$ & \\
\hline 9 & ETS_S & & ets $\{$ forecast $\}$, simulate $\{$ stats $\}$ & \\
\hline 10 & SES & Exponential smoothing & ses $\{$ forecast $\}$ & \\
\hline 11 & Theta & & thetaf $\{$ forecast $\}$ & \\
\hline
\end{tabular}




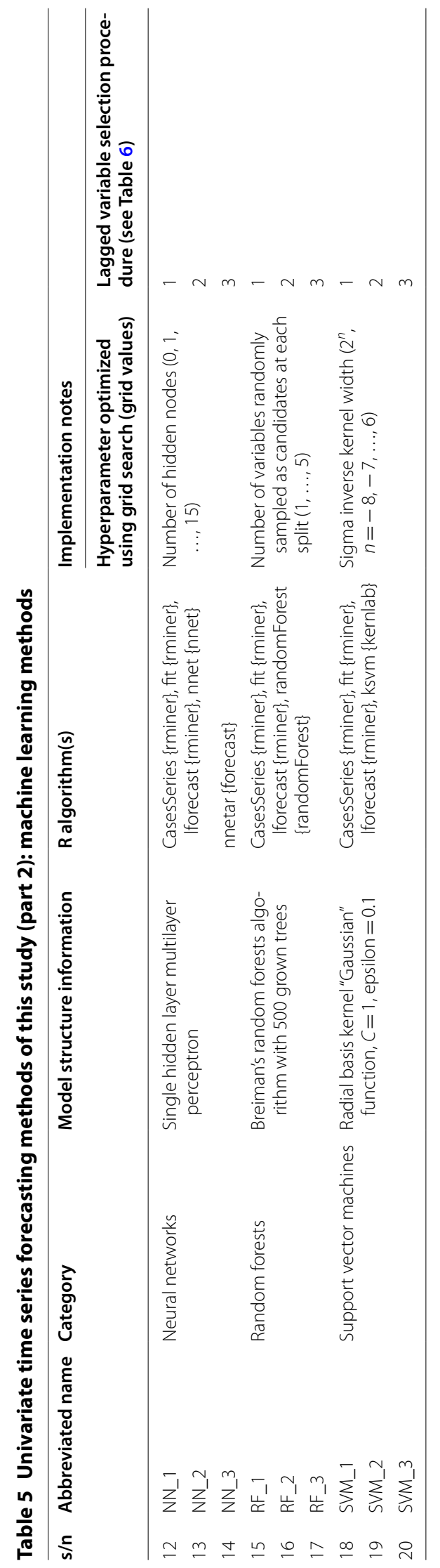


Table 6 Lagged variable selection procedures adopted for the machine learning methods of Table 5

\begin{tabular}{ll}
\hline $\mathbf{s} / \mathbf{n}$ & Time lags \\
\hline 1 & The corresponding to an estimated value for the AutoCorrelation Function (ACF) \\
2 & The corresponding to a statistical important estimated value for the ACF. If there is no statistical important estimated value for the acf $\{$ stats $\}$ \\
& ACF, the corresponding to the largest estimated value \\
According to nnetar $\{$ forecast $\}$, i.e. the time lags $1, \ldots, n$, where $n$ is the number of AutoRegressive (AR) parameters that are fitted to ar $\{$ stats $\}$ \\
the time series data
\end{tabular}

Table 7 Error metrics and accuracy statistics of this study

\begin{tabular}{|c|c|c|c|c|c|}
\hline $\mathrm{s} / \mathrm{n}$ & Abbreviated name & Full name & Category & Values & Optimum value \\
\hline 1 & $E$ & Error & Error metrics & $(-\infty,+\infty)$ & 0 \\
\hline 2 & $\mathrm{AE}$ & Absolute error & & {$[0,+\infty)$} & 0 \\
\hline 3 & PE & Percentage error & & $(-\infty,+\infty)$ & 0 \\
\hline 4 & APE & Absolute percentage error & & {$[0,+\infty)$} & 0 \\
\hline 5 & MdoAE & Median of the absolute errors & Accuracy statistics & {$[0,+\infty)$} & 0 \\
\hline 6 & MdoAPE & Median of the absolute percentage errors & & {$[0,+\infty)$} & 0 \\
\hline 7 & LRC & Linear regression coefficient & & $(-\infty,+\infty)$ & 1 \\
\hline 8 & $\mathrm{R} 2$ & Coefficient of determination & & {$[0,1]$} & 1 \\
\hline
\end{tabular}

Table 8 Experiments of this study

\begin{tabular}{|c|c|c|c|c|c|}
\hline$s / n$ & Abbreviated name & Category & Dataset (see Table 3) & Forecasting methods (see Tables 4 and 5) & Metrics (see Table 7) \\
\hline 1 & RWE_1i & Real-world & PrecDat & $1,2,7-20$ & $1-8$ \\
\hline 2 & RWE_2i & & TempDat & & \\
\hline 3 & RWE_3i & & StandPrecDat & $1,2,7-20$ & $1,2,5,7,8$ \\
\hline 4 & RWE_4i & & StandTempDat & & \\
\hline 5 & SE_1i & Simulation & SimDat_1 & $1-6,8-20$ & $1,2,5,7,8$ \\
\hline 6 & SE_2i & & SimDat_2 & & \\
\hline 7 & SE_3i & & SimDat_3 & & \\
\hline 8 & SE_4i & & SimDat_4 & & \\
\hline 9 & SE_5i & & SimDat_5 & & \\
\hline 10 & SE_6i & & SimDat_6 & & \\
\hline 11 & SE_7i & & SimDat_7 & & \\
\hline 12 & SE_8i & & SimDat_8 & $1,2,7-20$ & \\
\hline 13 & SE_9i & & SimDat_9 & & \\
\hline 14 & SE_10i & & SimDat_10 & & \\
\hline 15 & SE_11i & & SimDat_11 & & \\
\hline 16 & SE_12i & & SimDat_12 & & \\
\hline
\end{tabular}

The symbol $i$ can take the values stated in Table 9

approach. In particular, ARIMA_f, auto_ARIMA_f and auto_ARFIMA are theoretically expected to be the most accurate within our simulation experiments [for an explanation see Papacharalampous et al. (2017a), chapter 2], while BATS is also expected to perform well in these experiments, since it comprises an ARMA model. In summary, the experiments are controlled to some extent, while their components (datasets, methods and metrics) are selected to provide a multifaceted approach to the problem of one-step ahead forecasting in geoscience.

\section{Results and discussion}

In this section, we summarize the basic quantitative and qualitative information gained from the experiments of the present study, while the total amount is available in the Additional files 1, 2, 3, 4, 5, 6 and 7. We further 
Table 9 Part of the time series used within each experiment according to the $i$ value

\begin{tabular}{llll}
\hline $\mathbf{s} / \mathbf{n} \boldsymbol{i}$ & $\begin{array}{l}\text { Data points of each time series } \\
\text { used for the model-fitting (required } \\
\text { for all models) and model-validation } \\
\text { (required for the machine learning } \\
\text { models) }\end{array}$ & $\begin{array}{l}\text { Data points of each } \\
\text { time series used } \\
\text { for model-testing }\end{array}$ \\
\hline 1 & a $1,2,3, \ldots, 50$ & 51 \\
2 & b $1,2,3, \ldots, 60$ & 61 \\
3 & c $1,2,3, \ldots, 70$ & 71 \\
4 & d $1,2,3, \ldots, 80$ & 81 \\
5 & e $1,2,3, \ldots, 90$ & 91 \\
\hline
\end{tabular}

Table 10 Minimum, maximum and mean values of the MdoAE within the experiments using the PrecDat dataset

\begin{tabular}{llll}
\hline & Minimum $(\mathbf{m m})$ & Maximum $(\mathbf{m m})$ & Mean $(\mathbf{m m})$ \\
\hline RWE_1a & $111($ RF_1) & $172($ NN_1) & 135 \\
RWE_1b & $68($ SVM_1) & $146($ ETS_S $)$ & 91 \\
RWE_1C & 91 (SVM_3) & $171($ ETS_S $)$ & 119 \\
RWE_1d & 143 (BATS) & $189($ RF_2) & 162 \\
RWE_1e & 98 (Theta) & $150($ NN_1) & 122 \\
\hline
\end{tabular}

The minimum of the minimum values and the maximum of the maximum values are in italic

Table 11 Minimum, maximum and mean values of the MdoAPE within the experiments using the PrecDat dataset

\begin{tabular}{llll}
\hline & Minimum & Maximum & Mean \\
\hline RWE_1a & $0.12($ RF_1) & $0.21($ RW) & 0.16 \\
RWE_1b & 0.09(SVM_1) & $0.18($ ETS_s) & 0.12 \\
RWE_1c & $0.12($ SVM_3) & $0.21($ NN_1) & 0.15 \\
RWE_1d & $0.15($ BATS) & $0.22($ NN_1) & 0.17 \\
RWE_1e & 0.12 (Theta) & $0.18($ NN_1) & 0.15 \\
\hline
\end{tabular}

The minimum of the minimum values and the maximum of the maximum values are in italic

discuss the findings and explicate their contribution in light of the literature.

\section{Experiments using the precipitation datasets}

For the experiments using the PrecDat dataset, the minimum $\mathrm{AE}$ value is 0 (practically) and the maximum around $1,750 \mathrm{~mm}$ (for forecasts produced by the simple forecasting methods, i.e. Naïve and RW), while the respective values for the APE error metric are 0 (practically) and 1.64 (for a forecast produced by NN_1). The MdoAE and MdoAPE values are summarized in Tables 10 and 11 respectively. The minimum MdoAE is $68 \mathrm{~mm}$, while the maximum is $189 \mathrm{~mm}$. These two values are in the same order of magnitude as the smallest and average standard deviation estimates of the time series respectively. The minimum MdoAPE value is 0.09 and the maximum 0.22 , while the respective LRC values are 0.73 and 1.18 . The best LRC value (1.00) is measured within RWE_1c for the simple forecasting methods, while the best R2 value (0.84) is measured within RWE_1d for BATS. The worst LRC and R2 values are 0.73 for RF_2 within RWE_1d and 0.54 for NN_1 within RWE_1a respectively.

In Fig. 2 we present a graphical summary of the experiments using the PrecDat dataset. The values in the three upper heatmaps are scaled in the row direction and the darker the colour within a specific row the better the forecasts. In fact, heatmaps are used in this study instead of conventional tables, since they allow the easy extract of qualitative information. The relative performance of the forecasting methods differs to some degree across the various RWE_1 $i$ experiments, with ETS_s and NN_1 being the worst performing in terms of MdoAE and MdoAPE, followed by the simple methods. On the other hand, in terms of LRC Naïve and RW exhibit rather the best overall performance. In the downer heatmap of Fig. 2 we zoom into the RWE_1b experiment. By its examination we observe that all the implemented forecasting methods can perform well or bad, depending on the individual case. This fact is also apparent in the sideby-side boxplots of Fig. 2. Furthermore, we observe that for one specific time series the $\mathrm{AE}$ values measured are very high for all the forecasts apart from those produced by the simple forecasting methods.

Regarding the experiments using the StandPrecDat dataset, the minimum $\mathrm{AE}$ value is 0 and the maximum around 10. The MdoAE values are summarized in Table 12. The minimum MdoAE is 0.55 , while the maximum is 1.42 . These two values are $45 \%$ smaller and $42 \%$ larger than 1 (standard deviation of the standardized time series) respectively. Since there is an absence of relevant information in the literature, these values could be used as rough benchmarks for the predictability of annual precipitation. Most preferably, a representative sample set of univariate time series forecasting methods could be implemented at least for benchmarking purposes alongside with any other forecasting attempt. Moreover, the minimum and maximum LRC values are -0.25 and 0.25 respectively, the former measured for ETS_s and the latter for RW. Finally, the minimum R2 value is 0 (practically), while the maximum is 0.09 , measured within SE_3a for ETS_s. In addition to this numerical information, Fig. 3 presents a brief comparison between the experiments using the PrecDat and StandPrecDat datasets. As illustrated in this figure, the relative performance of the forecasting methods with respect to $\mathrm{AE}$ and MdoAE in the experiments using the latter dataset is mostly similar to the one in the experiments using 


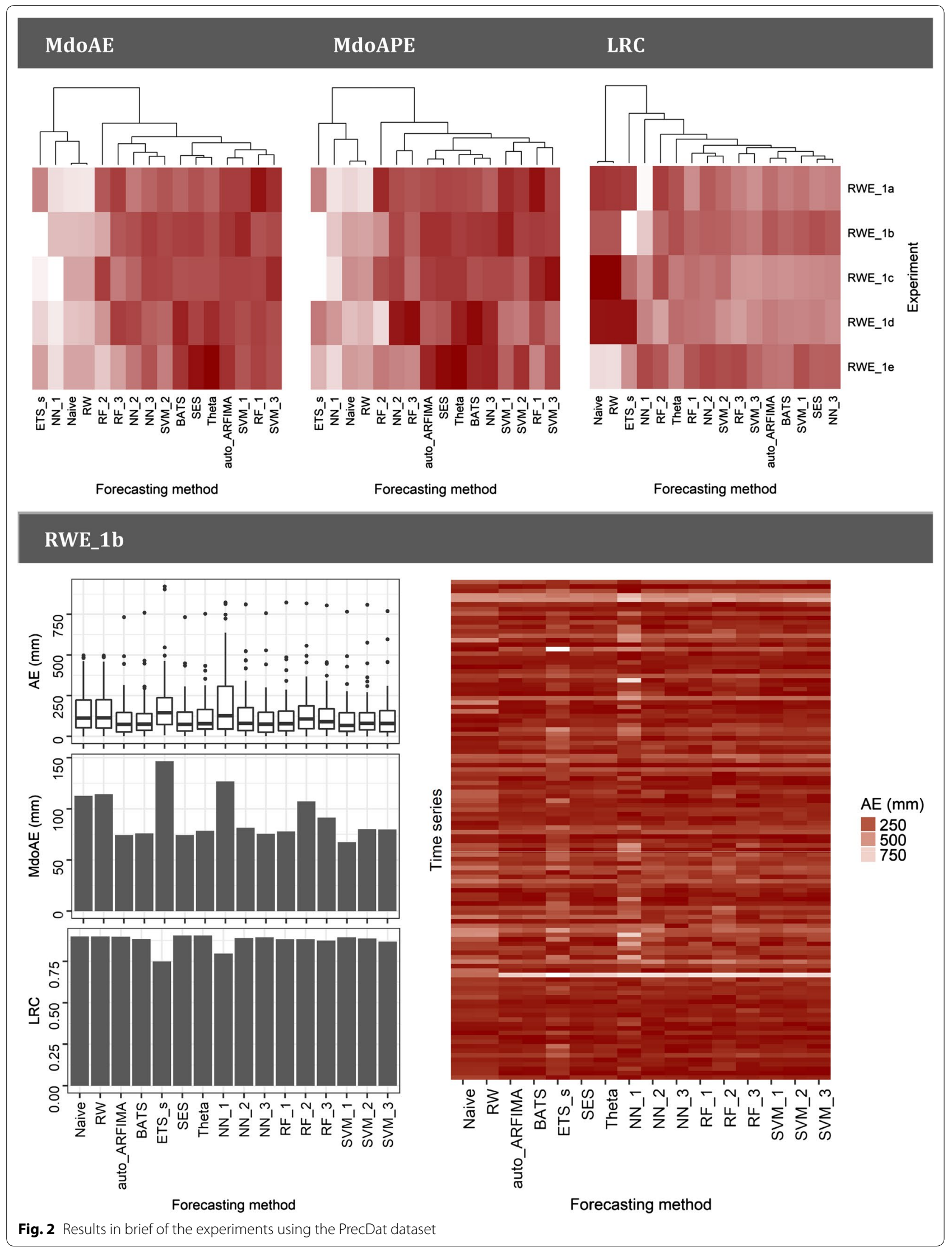


Table 12 Minimum, maximum and mean values of the MdoAE within the experiments using the StandPrecDat dataset

\begin{tabular}{llll}
\hline & Minimum & Maximum & Mean \\
\hline RWE_3a & $0.70($ RF_1) & $1.22($ NN_1) & 0.92 \\
RWE_3b & 0.55 (SVM_2) & $0.95($ ETS_S $)$ & 0.69 \\
RWE_3c & 0.72 (BATS) & $1.42($ NN_1) & 0.86 \\
RWE_3d & 0.99 (Theta) & $1.42($ ETS_S $)$ & 1.14 \\
RWE_3e & 0.69 (Theta) & 1.07 (ETS_s) & 0.89 \\
\hline
\end{tabular}

The minimum of the minimum values and the maximum of the maximum values are in italic

the former dataset. Nevertheless, the LRC (and R2) values are far worse when using the standardized datasets. In fact, standardization results to processes with different predictability with respect to the original.

\section{Experiments using the temperature datasets}

In Fig. 4 we present a graphical summary of the experiments using the TempDat dataset. For these experiments the minimum $\mathrm{AE}$ value is 0 and the maximum around $43{ }^{\circ} \mathrm{C}$ (for a forecast produced by $\mathrm{NN} \_2$ ), while the respective APE values are 0 and 9.64 (for a forecast produced by ETS_s). The MdoAE and MdoAPE values are summarized in Tables 13 and 14 respectively. The minimum MdoAE is $0.23{ }^{\circ} \mathrm{C}$, while the maximum is $1.10{ }^{\circ} \mathrm{C}$. These two values are in the same order of magnitude as the smallest and largest standard deviation estimates of the temperature time series respectively. The respective values for MdoAPE are 0.02 and 0.08 . The minimum LRC value is 0.95 and the maximum is 1.02; all the LRC values are close to the optimum. Finally, the minimum R2 value is 0.78 , measured for NN_2 within RWE_2b, while all the rest $\mathrm{R} 2$ values are higher than 0.97 with maximum 1 (practically), measured for the auto_ARFIMA method within RWE_2b. In summary, the relative performance of the forecasting methods varies across the different experiments conducted using the TempDat dataset. The auto_ARFIMA, BATS, SES, Theta and NN_3 seem to be well performing in terms of MdoAE and MdoAPE when applied to these temperature time series compared to the overall picture, while the simple methods are far the best in terms of MdoAE within the RWE_2d experiment. ETS_s and NN_1 are the worst performing within all the experiments apart from RWE_2c, in which the simple methods exhibit the worst performance. Finally, by comparing the numerical results of the experiments using the PrecDat and TempDat dataset, we observe the fact that temperature is more predictable than precipitation.

Regarding the experiments using the StandTempDat, the minimum $\mathrm{AE}$ value is 0 and the maximum around 18.91. The MdoAE values are summarized in Table 15 .
The minimum MdoAE value is 0.33 , while the maximum is 1.46 . These two values are $67 \%$ smaller and $46 \%$ larger than 1 (standard deviation of the standardized time series) respectively and could be used as rough benchmarks for the predictability of annual temperature (for an explanation see the subsection entitled "Experiments using the precipitation datasets"). The minimum LRC value is 0.04 and the maximum is 0.76 , the former measured for SVM_1 and the latter for RW. Finally, the minimum $\mathrm{R} 2$ value is 0.03 , while the maximum is 0.48 . The latter value is measured for Naïve in RWE_4a. Figure 5 facilitates a comparison between the experiments using the TempDat and StandTempDat datasets. Here as well, we observe that the relative performance of the forecasting methods with respect to $\mathrm{AE}$ and MdoAE in the experiments using the standardized precipitation time series mostly does not vary from the respective relative performance when using the original temperature time series. We further note that the LRC (and R2) values are worse when using the standardized temperature dataset, while they are better for the latter than for the standardized precipitation dataset.

\section{Experiments using the simulated datasets}

The subsequently reported information constitutes the provided empirical solution to the problem of one-step ahead forecasting in geoscience. Nonetheless, this solution is rather qualitative than quantitative (although the results are also stated quantitatively), since the respective experiments use unscaled data that could be assumed as realworld data in a standardized form (such as StandPrecDat and StandTempDat) with different predictability than the original (for example, see the subsections entitled "Experiments using the precipitation datasets" and "Experiments using the temperature datasets"). In fact, the experiments using standardized precipitation and temperature can facilitate a connection between the experiments using the same data in their original form and the experiments using the simulated datasets. A graphical summary of the latter experiments is available in Fig. 6.

The generalized findings of the present study are the following:

(1) The $E$ values are approximately symmetric around 0 (mean value of the simulations).

(2) The results may vary significantly across the simulation experiments using different simulated datasets and across the different time series within a specific experiment depending on the forecasting method.

(3) Consequently, the relative performance of the forecasting methods may also vary significantly across the simulation experiments using different simulated datasets. 


\section{Theta}

Experiment $\begin{aligned} & - \text { RWE_1a } \| \text { RWE_1c } \otimes R \text { RWE_1e } \\ & \Delta \text { RWE_1b }+ \text { RWE_1d }\end{aligned}$

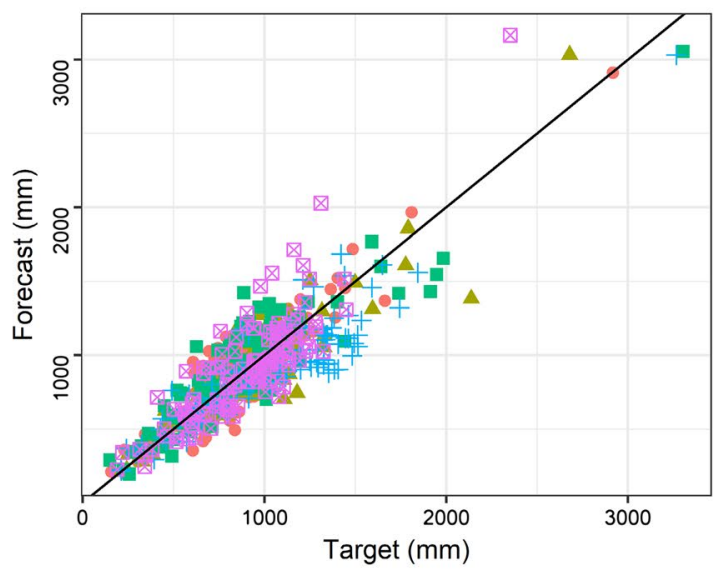

RWE_1d
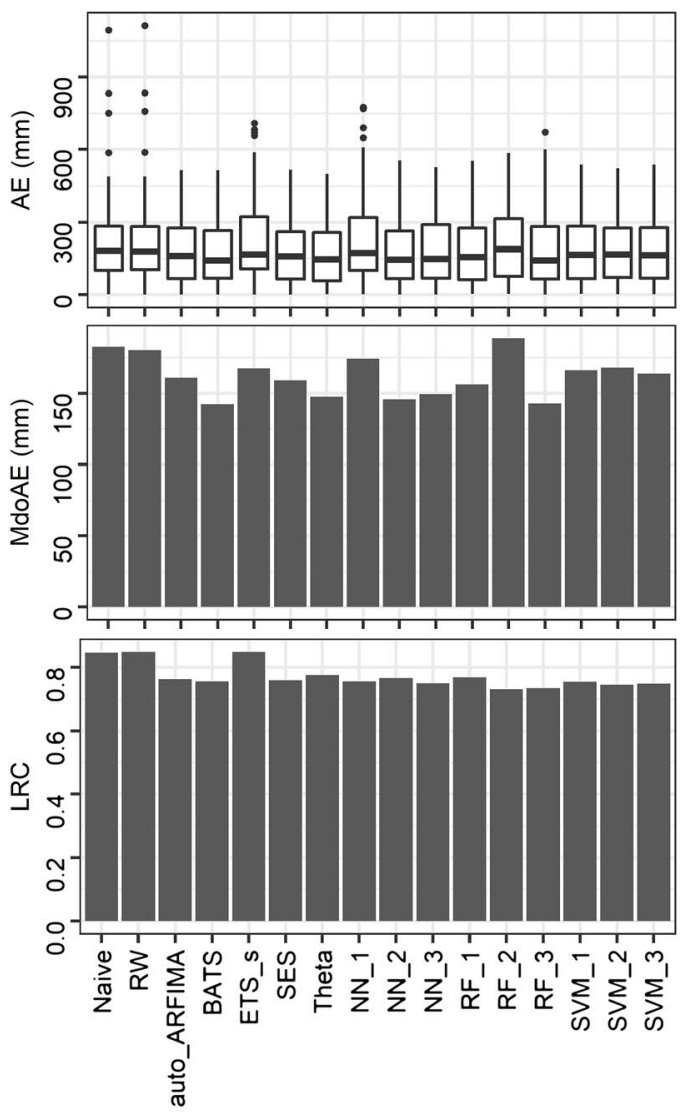

Forecasting method
Experiment - RWE_3a ॥ RWE_3c $₫$ RWE_3e $\Delta$ RWE_3b + RWE_3d

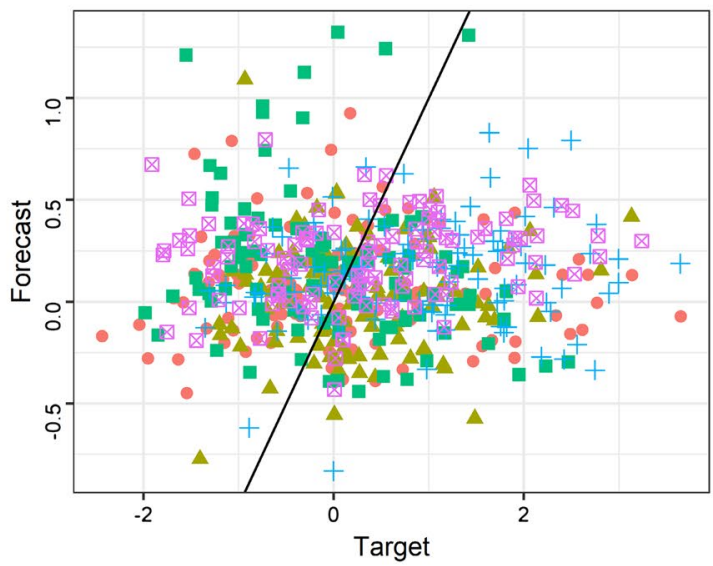

RWE_3d
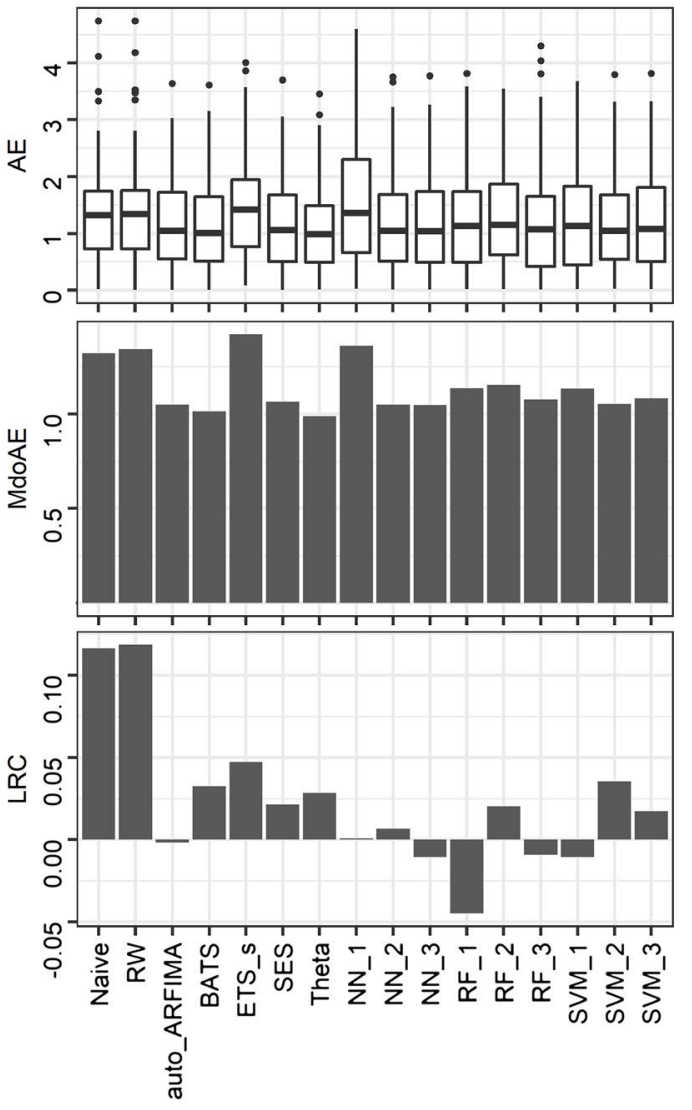

Forecasting method

Fig. 3 Comparison in brief between the experiments using the PrecDat and StandPrecDat datasets 


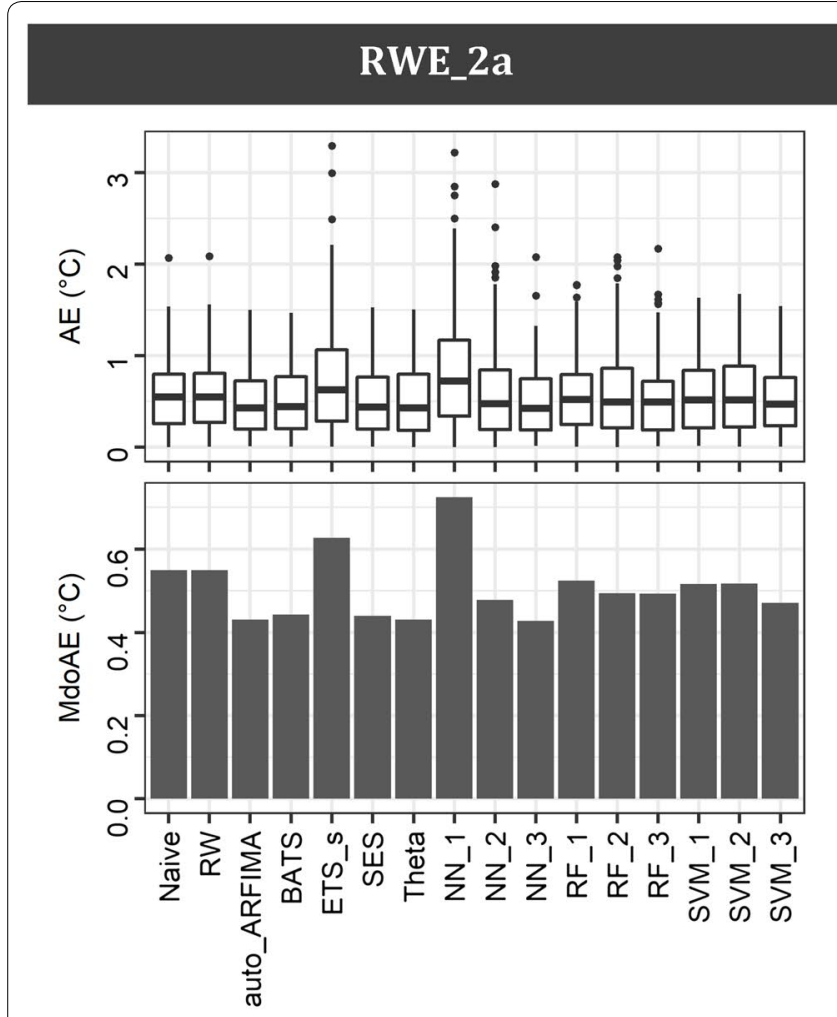

Forecasting method

\section{RWE_2c}
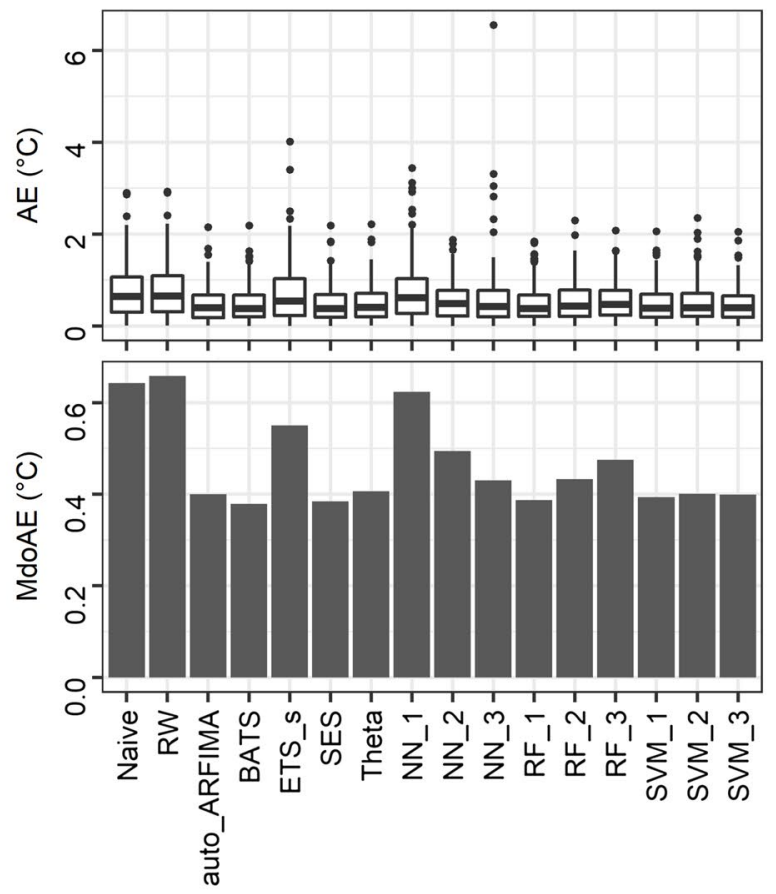

Forecasting method

\section{RWE_2d}
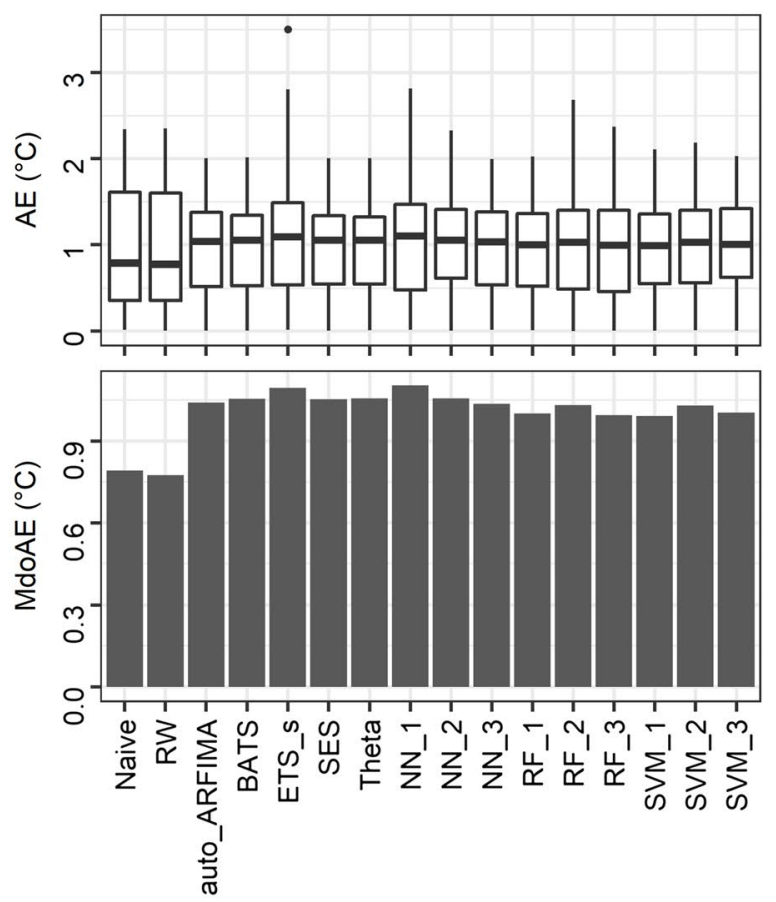

Forecasting method

Fig. 4 Results in brief of the experiments using the TempDat dataset
RWE_2e
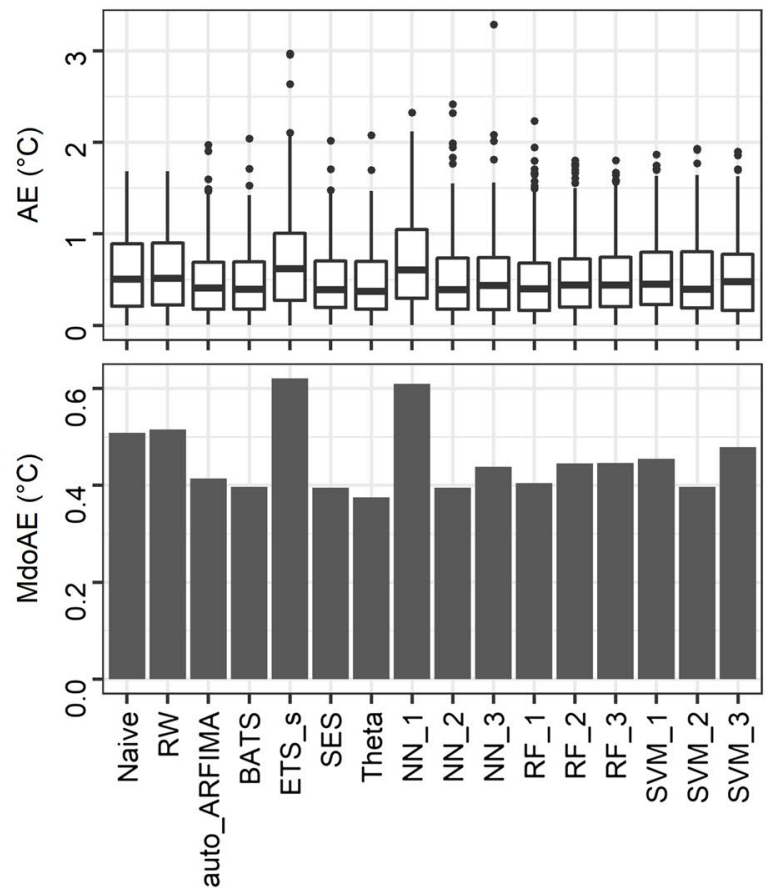

Forecasting method 
Table 13 Minimum, maximum and mean values of the MdoAE within the experiments using the TempDat dataset

\begin{tabular}{llll}
\hline & Minimum $\left({ }^{\circ} \mathrm{C}\right)$ & Maximum $\left({ }^{\circ} \mathrm{C}\right)$ & Mean $\left({ }^{\circ} \mathrm{C}\right)$ \\
\hline RWE_2a & $0.42($ NN_3) & $0.72\left(\mathrm{NN} \_1\right)$ & 0.51 \\
RWE_2b & $0.23($ Theta) & $0.54\left(\mathrm{NN} \_1\right)$ & 0.32 \\
RWE_2C & $0.38($ BATS) & $0.66(\mathrm{RW})$ & 0.47 \\
RWE_2d & $0.78($ RW $)$ & $1.10\left(\mathrm{NN} \_3\right)$ & 1.01 \\
RWE_2e & 0.38 (Theta) & $0.62($ ETS_S $)$ & 0.46 \\
\hline
\end{tabular}

The minimum of the minimum values and the maximum of the maximum values are in italic

Table 14 Minimum, maximum and mean values of the MdoAPE within the experiments using the TempDat dataset

\begin{tabular}{llll}
\hline & Minimum & Maximum & Mean \\
\hline RWE_2a & 0.04 (Theta) & $0.06($ NN_1) & 0.04 \\
RWE_2b & 0.02 (auto_ARFIMA) & $0.05($ ETS_s $)$ & 0.03 \\
RWE_2C & 0.03 (SVM_1) & $0.06($ RW) & 0.04 \\
RWE_2d & 0.07 (Naïve) & $0.08($ NN_1) & 0.08 \\
RWE_2e & 0.03 (RF_1) & $0.05($ NN_1) & 0.04 \\
\hline
\end{tabular}

The minimum of the minimum values and the maximum of the maximum values are in italic

Table 15 Minimum, maximum and mean values of the MdoAE within the experiments using the StandTempDat dataset

\begin{tabular}{llll}
\hline & Minimum & Maximum & Mean \\
\hline RWE_4a & 0.61 (BATS) & 0.93 (ETS_S) & 0.71 \\
RWE_4b & 0.33 (Theta) & $0.73($ NN_1) & 0.47 \\
RWE_4c & $0.56($ SES) & 0.96 (ETS_S) & 0.69 \\
RWE_4d & 1.20 (NN_1) & 1.46 (Theta) & 1.36 \\
RWE_4e & 0.48 (Theta) & 0.82 (ETS_s) & 0.61 \\
\hline
\end{tabular}

The minimum of the minimum values and the maximum of the maximum values are in italic

(4) On the contrary, the relative performance of the forecasting methods is slightly affected by the length of the time series for the experiments of the present study. The same has been found to mostly apply to the multi-step ahead forecasting performance of the same methods in Papacharalampous et al. (2017a) for two other time series lengths.

(5) Some forecasting methods are more accurate than others. The best-performing methods are ARIMA_f, auto_ARIMA_f, auto_ARFIMA, BATS, SES and Theta. This good performance of the former four methods when applied to ARMA and ARFIMA processes is expected from theory, while the Theta forecasting method has also performed well in the M3-Competition (Makridakis and Hibon 2000) and is expected to have a similar performance with SES (Hyndman and Billah 2003). The five above-mentioned forecasting methods are all stochastic.

(6) All the machine learning methods except for NN_1 (mostly NN_3 and SVM_3) are comparable to the best-performing methods, as it has also been found to apply in the experiments of Papacharalampous et al. (2017a, b). Likewise, in Tyralis and Papacharalampous (2017), random forests are competitive with the ARFIMA and Theta benchmarks.

(7) The simple methods are competitive in specific simulation experiments, as suggested for specific cases in Cheng et al. (2017), Makridakis and Hibon (2000) and Papacharalampous et al. (2017a) as well. Nevertheless, they also stand out because of their bad performance in other simulation experiments.

(8) Most of the far outliers are produced by neural networks.

The minimum $\mathrm{AE}$ value for the forecasts is 0 (practically) and the maximum around 155 (produced by NN_2). The MdoAE values are summarized in Tables 16 and 17. Especially, the latter is useful in supporting Observations (5-7). The minimum MdoAE is 0.65 , while the maximum is 2.91 . These two values are $35 \%$ smaller and $191 \%$ larger than 1 (standard deviation of the simulations) respectively. Furthermore, in spite of Observation (4), the MdoAE values may decrease on the level of the second or even the first decimal, when moving from the simulation experiments using time series of 51 values to those of 91 values, with the NN_1 forecasting method exhibiting the largest improvement. The minimum LRC value is -0.88 and the maximum is 0.94 , both measured for RW, while the minimum and maximum values produced by Naïve differ in the second and third decimal respectively. This range holds a complete interpretation of the observed within the real-world experiments variations in the performance of the simple methods in terms of LRC from extremely good to extremely bad (with respect to the overall picture). Finally, the minimum R2 value is 0 (practically), measured for ETS_s within several experiments, while the maximum is 0.84 within SE_9b for Naïve.

\section{Conclusions}

The simulation experiments reveal the most and least accurate methods for long-term one-step ahead forecasting applications, also suggesting that the simple methods may be competitive in specific cases. Furthermore, the relative performance of the forecasting methods is slightly affected by the time series length for the 


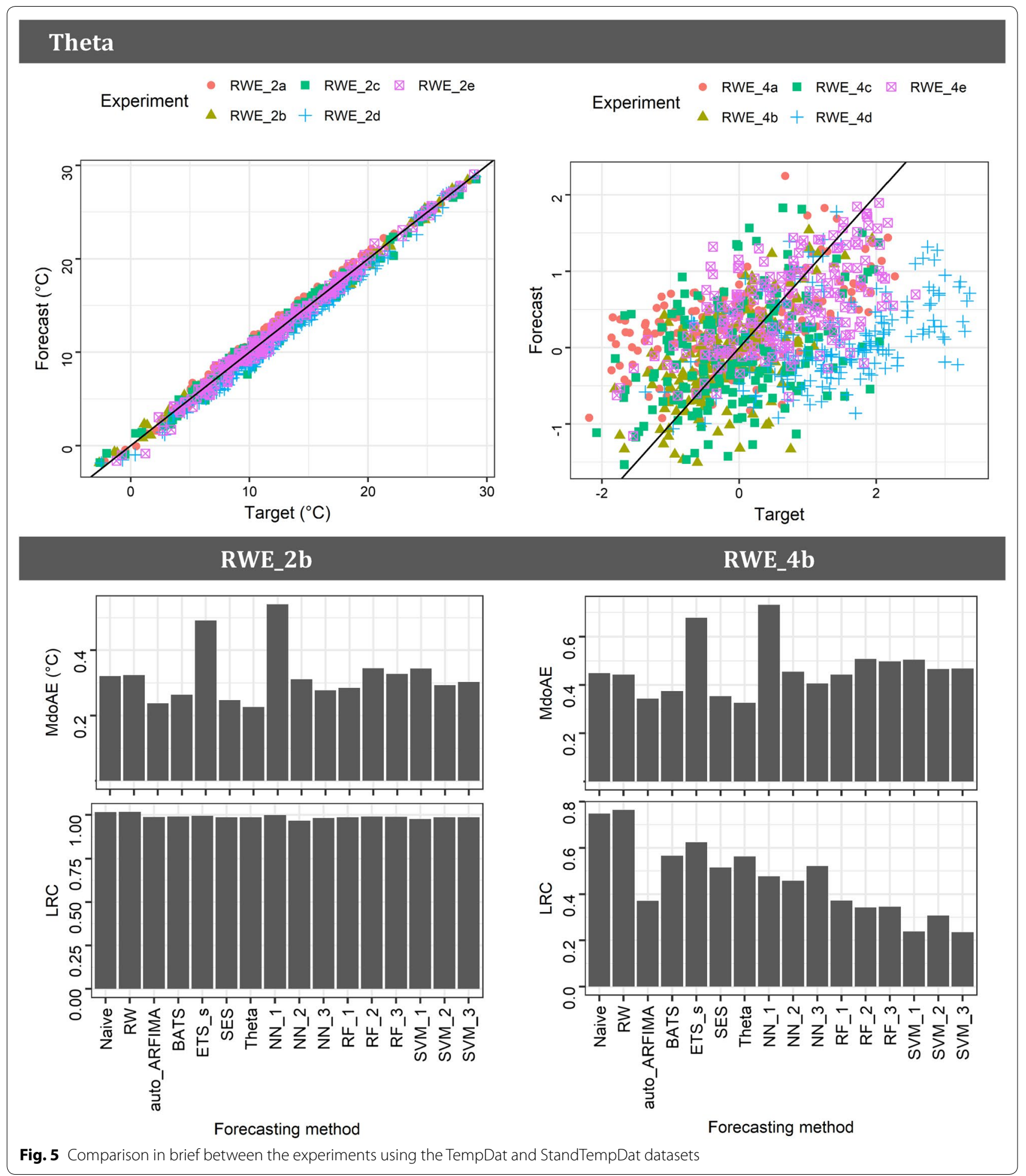

simulation experiments of this study (using time series of $51,61,71,81,91$ values), while it strongly depends on the process. Also importantly, the experiments using the original real-world time series result to minimum and maximum medians of the absolute errors of 68 and 189 $\mathrm{mm}$ for precipitation, and 0.23 and $1.10^{\circ} \mathrm{C}$ for temperature respectively. Additionally, the experiments using the standardized real-world time series suggest that the 

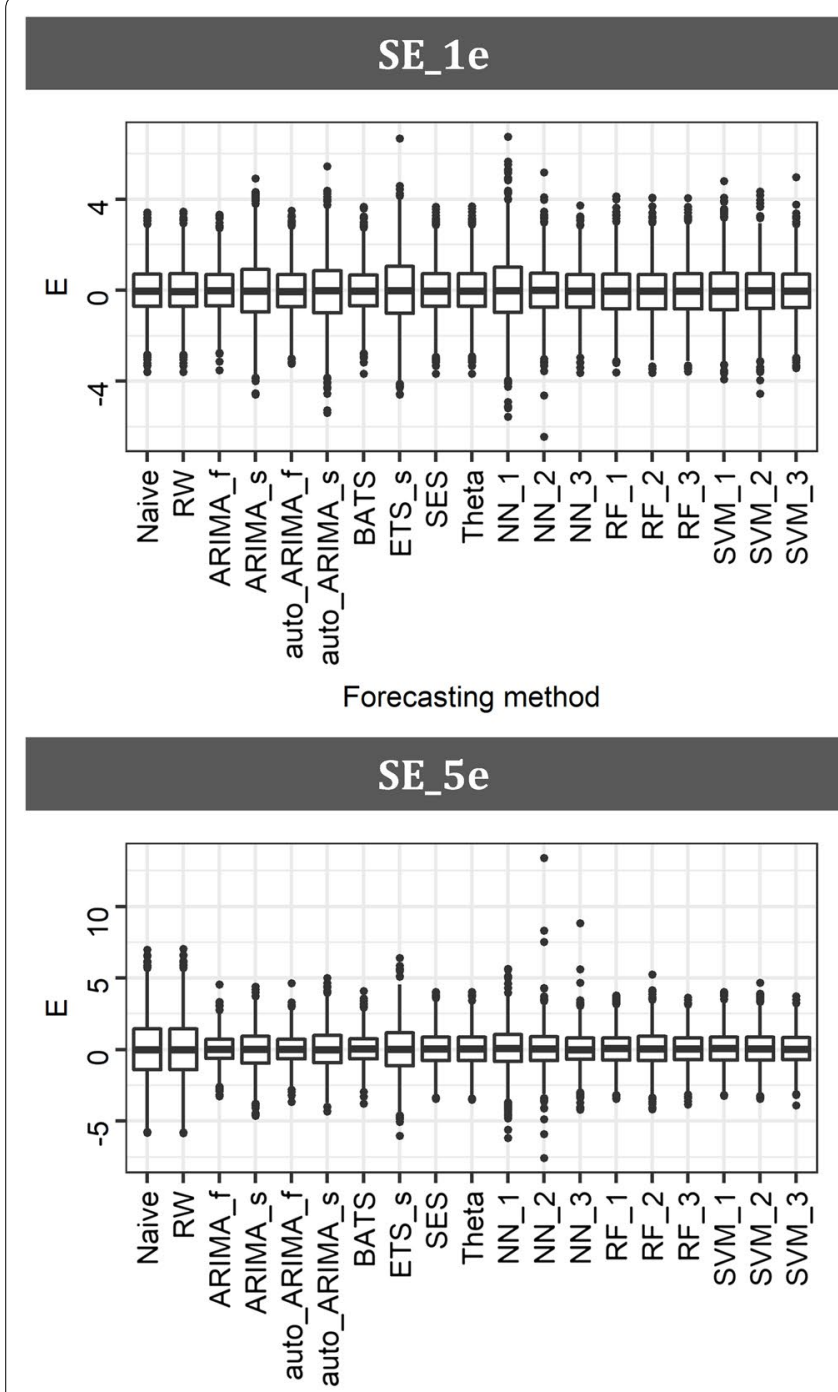

Forecasting method

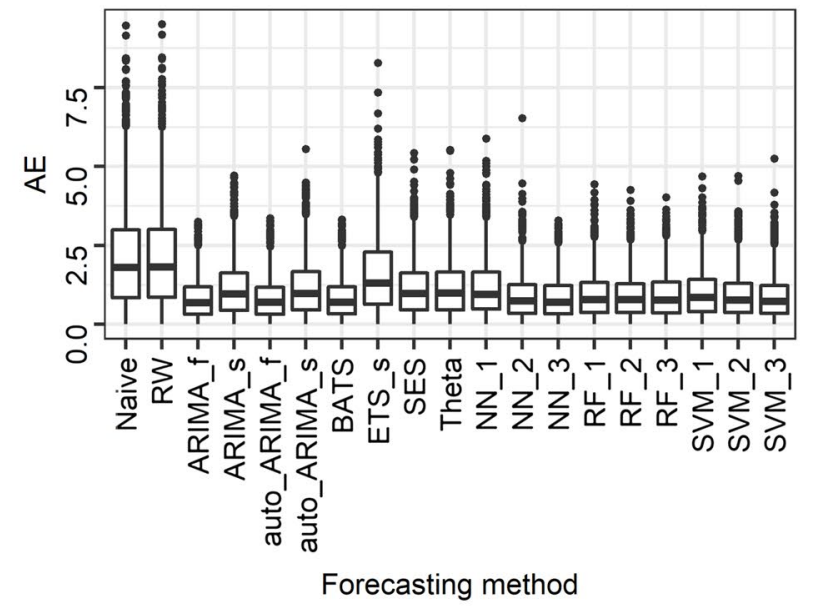

\section{SE_2e}

\section{SE_6e}

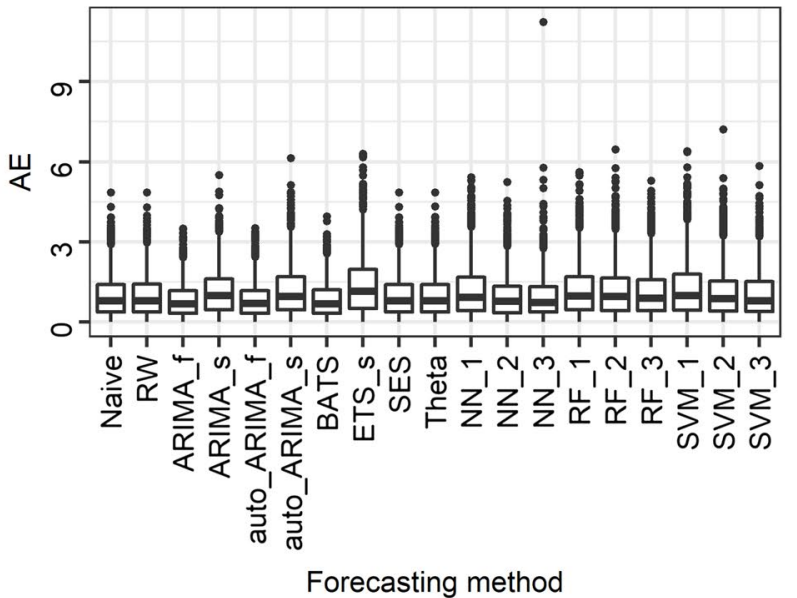

SE_9e

SE_11e

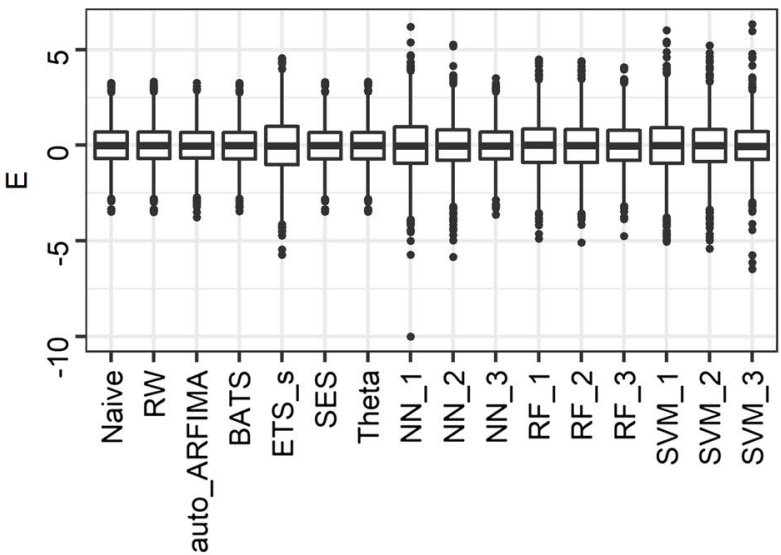

Forecasting method

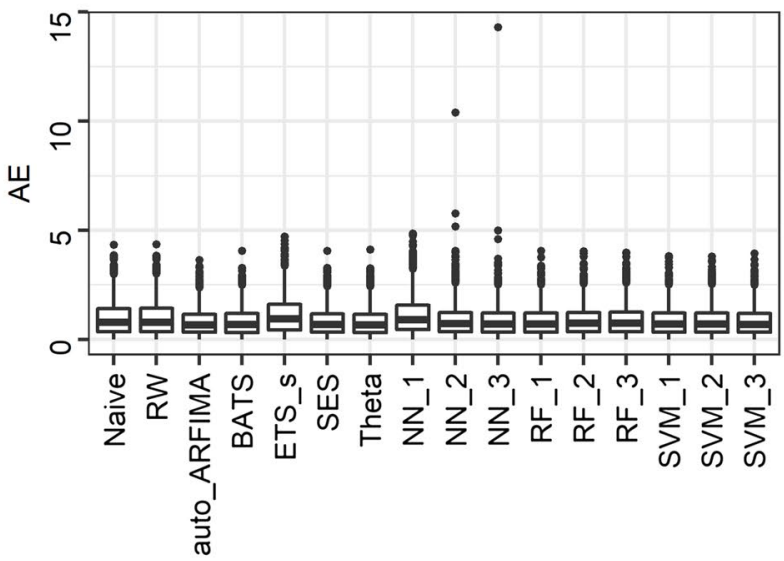

Forecasting method

Fig. 6 Results in brief of the experiments using the simulated datasets 
Table 16 Minimum, maximum and mean values of the MdoAE within the simulation experiments

\begin{tabular}{|c|c|c|c|}
\hline & Minimum & Maximum & Mean \\
\hline SE_1i & 0.68 (ARIMA_f $\mid$ SE_1a) & $1.05\left(N N \_1 \mid S E \_1 a\right)$ & 0.80 \\
\hline SE_2i & 0.67 (ARIMA_f $\mid S E \_2 C$ ) & $1.82\left(\mathrm{RW} \mid \mathrm{SE} \_2 \mathrm{e}\right)$ & 0.95 \\
\hline SE_3i & 0.65 (ARIMA_f $\mid$ SE_3C) & 1.04 (NN_1 | SE_3a) & 0.81 \\
\hline SE_4i & 0.67 (ARIMA_f $\mid S E \_4 C$ ) & 1.21 (ETS_s | SE_4a) & 0.84 \\
\hline SE_5i & 0.66 (ARIMA_f $\left.\mid S E \_5 e\right)$ & 1.48 (RW | SE_5c) & 0.90 \\
\hline SE_6i & 0.68 (ARIMA_f $\mid$ SE_6b) & 1.20 (ETS_s | SE_6d) & 0.89 \\
\hline SE_7i & 0.66 (auto_ARIMA_f |SE_7d) & 2.91 (RW | SE_7e) & 1.22 \\
\hline SE_8i & 0.67 (auto_ARFIMA |SE_8c) & $1.02\left(N N \_1 \mid S E \_8 a\right)$ & 0.77 \\
\hline SE_9i & 0.67 (auto_ARFIMA | SE_9d) & 1.05 (NN_1 | SE_9b) & 0.80 \\
\hline SE_10i & 0.67 (auto_ARFIMA |SE_10e) & 1.22 (RW | SE_10e) & 0.83 \\
\hline SE_11i & 0.68 (Theta |SE_11e) & $1.10\left(N N \_1 \mid\right.$ SE_11a) & 0.77 \\
\hline SE_12i & 0.69 (auto_ARFIMA | SE_12b) & 1.06 (NN_1 | SE_12a) & 0.78 \\
\hline
\end{tabular}

The minimum of the minimum values and the maximum of the maximum values are in italic

Table 17 Minimum, maximum and mean values of the MdoAE for each method within the simulation experiments

\begin{tabular}{|c|c|c|c|}
\hline & Minimum & Maximum & Mean \\
\hline Naïve & 0.68 (SE_3C) & 2.88 (SE_7a) & 1.12 \\
\hline RW & 0.69 (SE_9C) & 2.91 (SE_7e) & 1.13 \\
\hline ARIMA_f & 0.65 (SE_3C) & 0.72 (SE_7a) & 0.69 \\
\hline ARIMA_S & 0.91 (SE_2a) & 1.04 (SE_3a) & 0.96 \\
\hline auto_ARIMA_f & 0.66 (SE_7d) & 0.75 (SE_6C) & 0.70 \\
\hline auto_ARIMA_s & 0.91 (SE_4C) & 1.02 (SE_3d) & 0.97 \\
\hline auto_ARFIMA & 0.67 (SE_10e) & 0.73 (SE_10d) & 0.69 \\
\hline BATS & 0.67 (SE_3C) & 0.76 (SE_6C) & 0.71 \\
\hline ETS_S & $0.93\left(\mathrm{SE} \_3 d\right)$ & 2.11 (SE_7e) & 1.14 \\
\hline SES & 0.66 (SE_3C) & 1.52 (SE_7e) & 0.83 \\
\hline Theta & 0.66 (SE_3c) & 1.57 (SE_7a) & 0.84 \\
\hline NN_1 & 0.90 (SE_7e) & 1.16 (SE_7a) & 1.01 \\
\hline NN_2 & 0.72 (SE_8c) & 0.89 (SE_5b) & 0.79 \\
\hline NN_3 & 0.69 (SE_8c) & 0.84 (SE_6C) & 0.74 \\
\hline $\mathrm{RF}_{-} 1$ & 0.71 (SE_8C) & 1.08 (SE_6a) & 0.82 \\
\hline RF_2 & 0.72 (SE_8c) & 1.04 (SE_6C) & 0.83 \\
\hline RF_3 & 0.72 (SE_3C) & 0.98 (SE_6C) & 0.80 \\
\hline SVM_1 & 0.71 (SE_8e) & $1.23\left(\mathrm{SE} \_7 \mathrm{a}\right)$ & 0.86 \\
\hline SVM_2 & 0.68 (SE_8C) & 1.01 (SE_7a) & 0.81 \\
\hline SVM_3 & 0.68 (SE_8C) & 0.92 (SE_6C) & 0.76 \\
\hline
\end{tabular}

The minimum of the minimum values and the maximum of the maximum values are in italic

minimum and maximum medians of the absolute errors are 0.55 and 1.42 for precipitation, and 0.33 and 1.46 for temperature respectively. These latter numerical results could be used as a rough upper boundary for the one-step ahead predictability of annual precipitation and temperature.

We subsequently state the limitations of this study and some future directions. The provided empirical solution to the problem of one-step ahead forecasting in geoscience is rather qualitative than quantitative, while the experiments using standardized precipitation and temperature data have offered rough benchmarks only. In the future more real-world data could be used to develop improved benchmarks for assessing the respective predictabilities. It would be of interest to further investigate how these predictabilities depend on the location from which the data originate. In this case, more stations spanning around the globe would be required. Moreover, a direct and large-scale comparison, set on a common base (if this is feasible), between deterministic and statistical approaches to forecasting geophysical processes would be useful and interesting. Another limitation of this study is related to the adopted modelling approach, i.e. the datadriven one, according to which the selection of the model does not depend on the properties of the examined process and, therefore, the latter are mostly not investigated. Furthermore, the improvement of the performance of the machine learning models requires extensive comparisons between different procedures of hyperparameter optimization and lagged variable selection. Finally, future research could focus on the examination of the respective predictabilities, when using exogenous predictor variables as well, while a definitely worth-stated future direction is related to the adoption of probabilistic forecasting methods, instead of the point forecasting ones.

\section{Additional files}

\author{
Additional file 1. Exploration of the datasets. \\ Additional file 2. Experiments using the PrecDat dataset. \\ Additional file 3. Experiments using the TempDat dataset. \\ Additional file 4. Experiments using the StandPrecDat dataset. \\ Additional file 5. Experiments using the StandTempDat dataset. \\ Additional file 6. Experiments using the simulated datasets, part 1.
}

Additional file 7. Experiments using the simulated datasets, part 2.

\section{Abbreviations}

ACF: AutoCorrelation Function; AR: AutoRegressive; ARFIMA: AutoRegressive Fractionally Integrated Moving Average; ARIMA: AutoRegressive Integrated Moving Average; ARMA: AutoRegressive Moving Average; MA: Moving Average; SARIMA: Seasonal AutoRegressive Integrated Moving Average.

\section{Authors' contributions}

GP and HT worked on the analyses equally and to all of their aspects. GP, $\mathrm{HT}$ and DK discussed the results and contributed in the writing of the main manuscript. All authors read and approved the final manuscript. 


\section{Acknowledgements}

We thank the Editor Bellie Sivakumar and two anonymous reviewers, whose comments have substantially improved the quality of this paper.

The analyses and visualizations have been performed in R Programming Language (R Core Team 2017) by using the contributed R packages forecast (Hyndman and Khandakar 2008, Hyndman et al. 2017), fracdiff (Fraley et al. 2012), gdata (Warnes et al. 2017), ggplot2 (Wickham 2016), HKprocess (Tyralis 2016), kernlab (Karatzoglou et al. 2004), knitr (Xie 2014, 2015, 2017), nnet (Venables and Ripley 2002), randomForest (Liaw and Wiener 2002), readr (Wickham et al. 2017) and rminer (Cortez 2010, 2016).

We acknowledge the Asia Oceania Geoscience Society (AOGS) for providing the publication cost. A preliminary research by Papacharalampous et al. (2017d) was presented in the 14th AOGS Annual Meeting.

\section{Competing interests}

The authors declare that they have no competing interests.

\section{Availability of data and materials}

This is a fully reproducible research paper; all the codes and data, as well as their outcome results, are available in the Additional files (Papacharalampous and Tyralis 2018). The sources of the real-world datasets are Lawrimore et al. (2011) and Peterson and Vose (1997).

\section{Funding}

This research has not received any funding.

\section{Publisher's Note}

Springer Nature remains neutral with regard to jurisdictional claims in published maps and institutional affiliations.

Received: 31 August 2017 Accepted: 17 March 2018

Published online: 07 April 2018

\section{References}

Armstrong JS, Fildes R (2006) Making progress in forecasting. Int J Forecast 22(3):433-441. https://doi.org/10.1016/j.ijforecast.2006.04.007

Assimakopoulos V, Nikolopoulos K (2000) The theta model: a decomposition approach to forecasting. Int J Forecast 16(4):521-530. https://doi. org/10.1016/S0169-2070(00)00066-2

Babu CN, Reddy BE (2012) Predictive data mining on average global temperature using variants of ARIMA models. In: Proceeding of 2012 international conference on advances in engineering, science and management (ICAESM)

Ballini R, Soares S, Andrade MG (2001) Multi-step-ahead monthly streamflow forecasting by a neurofuzzy network model. In: IFSA World Congress and 20th NAFIPS International Conference, pp 992-997. https://doi. org/10.1109/nafips.2001.944740

Chau KW, Wu CL (2010) A hybrid model coupled with singular spectrum analysis for daily rainfall prediction. J Hydroinform 12(4):458-473. https://doi. org/10.2166/hydro.2010.032

Chawsheen TA, Broom M (2017) Seasonal time-series modeling and forecasting of monthly mean temperature for decision making in the Kurdistan Region of Iraq. J Stat Theory Pract 11(4):604-633. https://doi.org/10.1080/ 15598608.2017 .1292484

Chen XY, Chau KW, Busari AO (2015) A comparative study of population-based optimization algorithms for downstream river flow forecasting by a hybrid neural network model. Eng Appl Artif Intell 46(Part A):258-268. https://doi.org/10.1016/j.engappai.2015.09.010

Cheng KS, Lien YT, Wu YC, Su YF (2017) On the criteria of model performance evaluation for real-time flood forecasting. Stoch Environ Res Risk Assess 31(5):1123-1146. https://doi.org/10.1007/s00477-016-1322-7

Cortez P (2010) Data mining with neural networks and support vector machines using the R/rminer tool. In: Perner P (ed) Advances in data mining. Applications and theoretical aspects. Springer, Heidelberg, pp 572-583. https://doi.org/10.1007/978-3-642-14400-4 44
Cortez P (2016) rminer: data mining classification and regression methods. $R$ package version 1.4.2. https://CRAN.R-project.org/package=rminer

De Gooijer JG, Hyndman RJ (2006) 25 years of time series forecasting. Int J Forecast 22(3):443-473. https://doi.org/10.1016/j.ijforecast.2006.01.001

De Livera AM, Hyndman RJ, Snyder RS (2011) Forecasting time series with complex seasonal patterns using exponential smoothing. J Am Stat Assoc 106(496):1513-1527. https://doi.org/10.1198/jasa.2011.tm09771

Fildes R, Kourentzes N (2011) Validation and forecasting accuracy in models of climate change. Int J Forecast 27(4):968-995. https://doi.org/10.1016/j. ijforecast.2011.03.008

Fraley C, Leisch F, Maechler M, Reisen V, Lemonte A (2012) fracdiff: fractionally differenced ARIMA aka ARFIMA(p,d,q) models. R package version 1.4-2. https://CRAN.R-project.org/package $=$ fracdiff

Gholami V, Chau KW, Fadaee F, Torkaman J, Ghaffari A (2015) Modeling of groundwater level fluctuations using dendrochronology in alluvial aquifers. J Hydrol 529(Part 3):1060-1069. https://doi.org/10.1016/] jhydrol.2015.09.028

Giunta G, Salerno R, Ceppi A, Ercolani G, Mancini M (2015) Benchmark analysis of forecasted seasonal temperature over different climatic areas. Geosc Lett 2:9. https://doi.org/10.1186/s40562-015-0026-z

Green KC, Armstrong JS (2007) Global warming: forecasts by scientists versus scientific forecasts. Energy Environ 18(7):997-1021. https://doi. org/10.1260/095830507782616887

Green KC, Armstrong JS, Soon W (2009) Validity of climate change forecasting for public policy decision making. Int J Forecast 25(4):826-832. https:// doi.org/10.1016/j.ijforecast.2009.05.011

Hong WC (2008) Rainfall forecasting by technological machine learning models. Appl Math Comput 200(1):41-57. https://doi.org/10.1016/j. amc.2007.10.046

Htike KK, Khalifa OO (2010) Rainfall forecasting models using focused timedelay neural networks. In: Proceeding of 2010 international conference on computer and communication engineering (ICCCE). https://doi. org/10.1109/iccce.2010.5556806

Hyndman RJ, Athanasopoulos G (2013) Forecasting: principles and practice. OTexts: Melbourne, Australia. http://otexts.org/fpp/

Hyndman RJ, Billah B (2003) Unmasking the Theta method. Int J Forecasting 19(2):287-290. https://doi.org/10.1016/S0169-2070(01)00143-1

Hyndman RJ, Khandakar Y (2008) Automatic time series forecasting: the forecast package for R. J Stat Softw 27(3):1-22. https://doi.org/10.18637/ jss.v027.i03

Hyndman RJ, O'Hara-Wild M, Bergmeir C, Razbash S, Wang E (2017) forecast: forecasting functions for time series and linear models. R package version 8.2. https://CRAN.R-project.org/package=forecast

Karatzoglou A, Smola A, Hornik K, Zeileis A (2004) kernlab-an S4 package for kernel methods in R. J Stat Softw 11(9):1-20

Keenlyside NS (2011) Commentary on "Validation and forecasting accuracy in models of climate change". Int J Forecast 27(4):1000-1003. https://doi. org/10.1016/j.ijforecast.2011.07.002

Komorník J, Komorníková M, Mesiar R, Szökeová D, Szolgay J (2006) Comparison of forecasting performance of nonlinear models of hydrological time series. Phys Chem Earth Parts A/B/C 31(18):1127-1145. https://doi. org/10.1016/j.pce.2006.05.006

Koutsoyiannis D (2008) Probability and statistics for geophysical processes. National Technical University of Athens, Athens. https://doi.org/10.13140/ RG.2.1.2300.1849/1

Koutsoyiannis D, Yao H, Georgakakos A (2008) Medium-range flow prediction for the Nile: a comparison of stochastic and deterministic methods. Hydrol Sci J 53(1):142-164. https://doi.org/10.1623/hysj.53.1.142

Lambrakis N, Andreou AS, Polydoropoulos P, Georgopoulos E, Bountis T (2000) Nonlinear analysis and forecasting of a brackish karstic spring. Water Resour Res 36(4):875-884. https://doi.org/10.1029/1999WR900353

Lawrimore JH, Menne MJ, Gleason BE, Williams CN, Wuertz DB, Vose RS, Rennie J (2011) An overview of the Global Historical Climatology Network monthly mean temperature data set, version 3. J Geophys Res. https:// doi.org/10.1029/2011JD016187

Liaw A, Wiener M (2002) Classification and regression by randomForest. $R$ News 2(3):18-22

Makridakis S, Hibon M (2000) The M3-competition: results, conclusions and implications. Int J Forecast 16(4):451-476. https://doi.org/10.1016/ S0169-2070(00)00057-1 
McSharry PE (2011) Validation and forecasting accuracy in models of climate change: comments. Int J Forecast 27(4):996-999. https://doi. org/10.1016/j.ijforecast.2011.07.003

Narayanan P, Basistha A, Sarkar S, Kamna S (2013) Trend analysis and ARIMA modelling of pre-monsoon rainfall data for western India. C R Geosci 345(1):22-27. https://doi.org/10.1016/j.crte.2012.12.001

Papacharalampous GA, Tyralis H (2018) One-step ahead forecasting of geophysical processes within a purely statistical framework: supplementary material. figshare. https://doi.org/10.6084/m9.figshare.5357359.v1

Papacharalampous GA, Tyralis H, Koutsoyiannis D (2017a) Comparison of stochastic and machine learning methods for the multi-step ahead forecasting of hydrological processes. Preprints. https://doi.org/10.20944/ preprints201710.0133.v1

Papacharalampous GA, Tyralis H, Koutsoyiannis D (2017b) Error evolution in multi-step ahead streamflow forecasting for the operation of hydropower reservoirs. Preprints. https://doi.org/10.20944/preprints201710.0129.v1

Papacharalampous GA, Tyralis H, Koutsoyiannis D (2017c) Forecasting of geophysical processes using stochastic and machine learning algorithms. Eur Water 59:161-168

Papacharalampous GA, Tyralis H, Koutsoyiannis D (2017d) Large scale simulation experiments for the assessment of one-step ahead forecasting properties of stochastic and machine learning point estimation methods. Asia Oceania Geosciences Society (AOGS) 14th Annual Meeting, Singapore. http://www.itia.ntua.gr/en/docinfo/1719/

Papacharalampous GA, Tyralis H, Koutsoyiannis D (2018) Predictability of monthly temperature and precipitation using automatic time series forecasting methods. Acta Geophys. https://doi.org/10.1007/ s11600-018-0120-7

Peterson TC, Vose RS (1997) An Overview of the Global Historical Climatology Network temperature database. B Am Meteorol Soc. 78:2837-2849. https://doi.org/10.1175/1520-0477(1997)078<2837:AOOTGH>2.0.CO;2

R Core Team (2017) R: a language and environment for statistical computing. $R$ Foundation for Statistical Computing, Vienna

Remesan R, Mathew J (2015) Hydrological data driven modelling. Springer International Publishing, New York. https://doi. org/10.1007/978-3-319-09235-5

Sivakumar B (2017) Chaos in hydrology: bridging determinism and stochasticity. Springer, New York. https://doi.org/10.1007/978-90-481-2552-4

Taormina R, Chau KW (2015) Data-driven input variable selection for rainfallrunoff modeling using binary-coded particle swarm optimization and extreme learning machines. J Hydrol 529(Part 3):1617-1632. https://doi. org/10.1016/j.jhydrol.2015.08.022

Tyralis H (2016) HKprocess: Hurst-Kolmogorov process. R package version 0.02. https://CRAN.R-project.org/package $=$ HKprocess

Tyralis H, Koutsoyiannis D (2011) Simultaneous estimation of the parameters of the Hurst-Kolmogorov stochastic process. Stoch Environ Res Risk Assess 25(1):21-33. https://doi.org/10.1007/s00477-010-0408-x
Tyralis H, Koutsoyiannis D (2014) A Bayesian statistical model for deriving the predictive distribution of hydroclimatic variables. Clim Dyn 42(1112):2867-2883. https://doi.org/10.1007/s00382-013-1804-y

Tyralis H, Koutsoyiannis D (2017) On the prediction of persistent processes using the output of deterministic models. Hydrol Sci J 62(13):2083-2102

Tyralis H, Papacharalampous G (2017) Variable selection in time series forecasting using random forests. Algorithms 10(4):114. https://doi.org/10.3390/ a10040114

Venables WN, Ripley BD (2002) Modern applied statistics with S, 4th edn. Springer-Verlag, New York. https://doi.org/10.1007/978-0-387-21706-2

Wang S, Feng J, Liu G (2013) Application of seasonal time series model in the precipitation forecast. Math Comput Model 58(3-4):677-683. https://doi. org/10.1016/j.mcm.2011.10.034

Wang W, Chau K, Xu D, Chen XY (2015) Improving forecasting accuracy of annual runoff time series using ARIMA based on EEMD decomposition. Water Resour Manag 29(8):2655-2675. https://doi.org/10.1007/ s11269-015-0962-6

Warnes GR, Bolker B, Gorjanc G, Grothendieck G, Korosec A, Lumley T, MacQueen D, Magnusson A, Rogers J et al (2017) gdata: various R programming tools for data manipulation. R package version 2.18.0. https:// CRAN.R-project.org/package $=$ gdata

Wei WWS (2006) Time series analysis, univariate and multivariate methods, 2nd edn. Pearson Addison Wesley, Boston

Wickham H (2016) ggplot2: elegant graphics for data analysis, 2nd edn. Springer International Publishing, Cham. https://doi. org/10.1007/978-3-319-24277-4

Wickham H, Hester J, Francois R, Jylänki J, Jørgensen M (2017) readr: read rectangular text data. R package version 1.1.1. https://CRAN.R-project. org/package $=$ readr

Wu CL, Chau KW, Fan C (2010) Prediction of rainfall time series using modular artificial neural networks coupled with data-preprocessing techniques. J Hydrol 389(1-2):146-167. https://doi.org/10.1016/j.jhydrol.2010.05.040

Xie Y (2014) knitr: a comprehensive tool for reproducible research in $\mathrm{R}$. In: Stodden V, Leisch F, Peng RD (eds) Implementing reproducible computational research. Chapman and Hall/CRC, Boca Raton

Xie Y (2015) Dynamic documents with R and knitr, 2nd edn. Chapman and Hall/CRC, Boca Raton

Xie Y (2017) knitr: a general-purpose package for dynamic report generation in R. R package version 1.17. https://CRAN.R-project.org/package=knitr

Yu X, Liong SY (2007) Forecasting of hydrologic time series with ridge regression in feature space. J Hydrol 332(3-4):290-302. https://doi. org/10.1016/j.jhydrol.2006.07.003

Yu X, Liong SY, Babovic V (2004) EC-SVM approach for real-time hydrologic forecasting. J Hydroinform 6(3):209-223

\section{Submit your manuscript to a SpringerOpen ${ }^{\circ}$ journal and benefit from:}

- Convenient online submission

- Rigorous peer review

- Open access: articles freely available online

- High visibility within the field

- Retaining the copyright to your article

Submit your next manuscript at springeropen.com 